\title{
A proangiogenic signaling axis in myeloid cells promotes malignant progression of glioma
}

\author{
Yujie Huang, ${ }^{1}$ Prajwal Rajappa, ${ }^{1}$ Wenhuo Hu, ${ }^{2}$ Caitlin Hoffman, ${ }^{1}$ Babacar Cisse, ${ }^{1}$ Joon-Hyung Kim, ${ }^{1}$ Emilie Gorge,' Rachel Yanowitch, \\ William Cope, ${ }^{1}$ Emma Vartanian, ${ }^{1}$ Raymond Xu, ${ }^{1}$ Tuo Zhang, ${ }^{3}$ David Pisapia, ${ }^{4}$ Jenny Xiang, ${ }^{3}$ Jason Huse, ${ }^{2}$ Irina Matei, ${ }^{5}$ \\ Hector Peinado, Jacqueline Bromberg, ${ }^{6}$ Eric Holland, ${ }^{7}$ Bi-sen Ding, ${ }^{8}$ Shahin Rafii, ${ }^{8}$ David Lyden, ${ }^{5}$ and Jeffrey Greenfield \\ 'Children's Brain Tumor Project, Department of Neurological Surgery, Weill Cornell Medicine, New York, New York, USA. ${ }^{2}$ Human Oncology and Pathogenesis Program, Memorial Sloan Kettering Cancer Center, \\ New York, New York, USA. ${ }^{3}$ Department of Microbiology and Immunology, ${ }^{4}$ Department of Pathology, and ${ }^{5}$ Children's Cancer and Blood Foundation Laboratories, Departments of Pediatrics, and Cell and \\ Developmental Biology, Drukier Institute for Children's Health, Meyer Cancer Center, Weill Cornell Medicine, New York, New York, USA. ${ }^{6}$ Department of Medicine, Memorial Sloan Kettering Cancer Center, New \\ York, New York, USA. Division of Human Biology, Solid Tumor and Translational Research, Fred Hutchinson Cancer Research Center, Seattle, Washington, USA. ${ }^{8}$ Department of Cenetic Medicine, Weill Cornell \\ Medicine, New York, New York, USA
}

Tumors are capable of coopting hematopoietic cells to create a suitable microenvironment to support malignant growth. Here, we have demonstrated that upregulation of kinase insert domain receptor (KDR), also known as VEGFR2, in a myeloid cell sublineage is necessary for malignant progression of gliomas in transgenic murine models and is associated with high-grade tumors in patients. KDR expression increased in myeloid cells as myeloid-derived suppressor cells (MDSCs) accumulated, which was associated with the transformation and progression of low-grade fibrillary astrocytoma to high-grade anaplastic gliomas. KDR deficiency in murine BM-derived cells (BMDCs) suppressed the differentiation of myeloid lineages and reduced granulocytic/monocytic populations. The depletion of myeloid-derived KDR compromised its proangiogenic function, which inhibited the angiogenic switch necessary for malignant progression of low-grade to high-grade tumors. We also identified inhibitor of DNA binding protein 2 (ID2) as a key upstream regulator of KDR activation during myeloid differentiation. Deficiency of ID2 in BMDCs led to downregulation of KDR, suppression of proangiogenic myeloid cells, and prevention of low-grade to high-grade transition. Tumor-secreted TCF- $\beta$ and granulocyte-macrophage CSF (GM-CSF) enhanced the KDR/ ID2 signaling axis in BMDCs. Our results suggest that modulation of KDR/ID2 signaling may restrict tumor-associated myeloid cells and could potentially be a therapeutic strategy for preventing transformation of premalignant gliomas.

\section{Introduction}

The emerging role of BM-derived cells (BMDCs), including myeloid-derived suppressor cells (MDSCs), dendritic cells, neutrophils, and macrophages (1-6), has become a focus of recent research in malignant progression. Numerous studies have demonstrated that tumors directly influence hematopoietic cells toward protumoral phenotypes via complex molecular signaling pathways $(4,7)$. Interactions with stromal components or tumor cells allow BMDCs to promote angiogenesis, resistance, and invasion in various types of cancers, including brain tumors $(5,8)$.

Gliomas represent the most prevalent malignant brain tumors and are classified by WHO as grade I, II, III, or IV. WHO grades are uniquely defined by the presence of specific pathological features, including cell proliferation, necrosis, and the extent of angiogenesis (9). In comparison with their low-grade (grade II) counterparts, highgrade gliomas (grade III/IV) are characterized by microvascular proliferation and necrosis (9-11). While a percentage of high-grade gliomas develop de novo, these tumors may also result from malignant transformation of lower grade tumor variants $(9,10,12)$. Patients with

Conflict of interest: The authors have declared that no conflict of interest exists. Submitted: January 11, 2016; Accepted: February 16, 2017.

Reference information: / Clin Invest. 2017;127(5):1826-1838.

https://doi.org/10.1172/JCl86443. low-grade gliomas have an increased chance of cure using aggressive therapies, including total surgical resection and chemotherapy, and may live many progression-free years with residual tumor that cannot safely be removed through gross total resection $(10,12,13)$. Once lowgrade tumors undergo malignant transformation to a higher grade, however, prognosis often mirrors de novo diagnosis of high-grade gliomas and often leads to poor clinical outcomes $(14,15)$.

A more thorough understanding of the tumor microenvironment, particularly the role of BMDCs towards malignant glioma transformation, has substantial biological and clinical relevance insofar as arresting malignant progression has implications for long-term disease survival. In this study, we found that kinase insert domain receptor (KDR), also known as VEGFR2, is responsible for driving differentiation of hematopoietic progenitor cells (HPCs) into protumoral MDSCs. Through enhanced signaling of hematopoieticderived inhibitor of DNA binding protein 2 (ID2), a member of the inhibitor of DNA binding proteins class, primary tumors direct myeloid progenitors to develop into tumor-associated BMDCs via upregulation of KDR and, in turn, stimulate the aggressive proangiogenic phenotype of gliomas.

\section{Results}

Intracellular KDR in glioma-associated myeloid cells. KDR has been identified in both endothelial and endothelial progenitor cells 
A

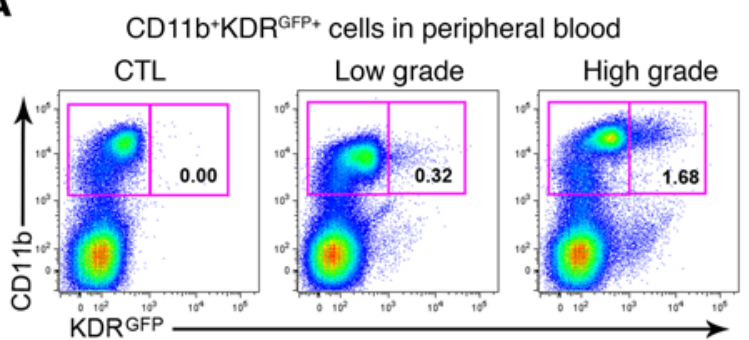

B

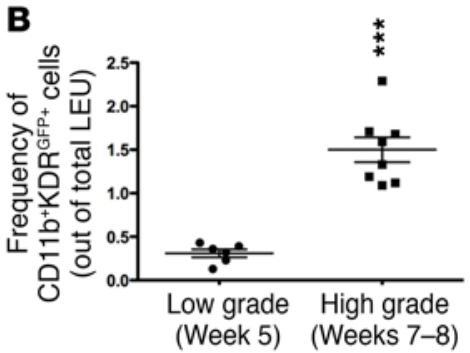

c

Monocytic/granulocytic cells in peripheral blood

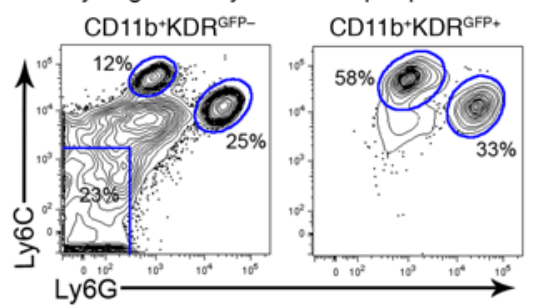

E

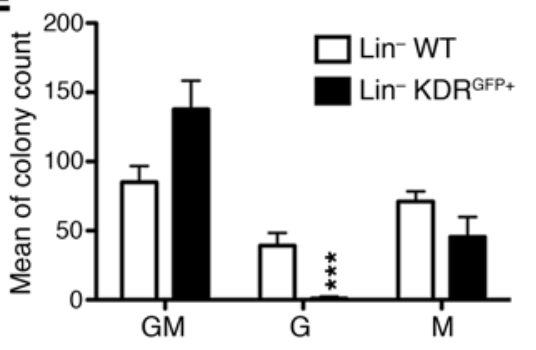

D

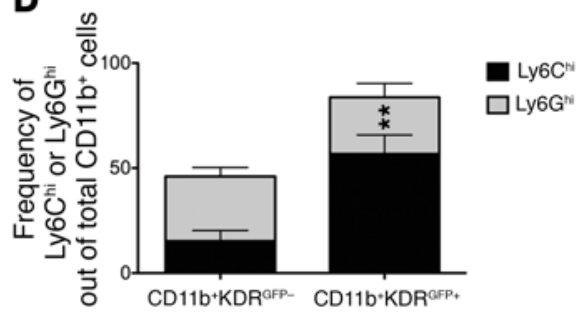

F

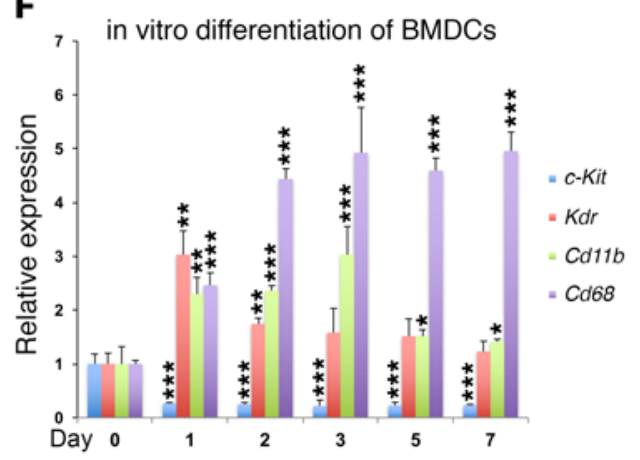

Figure 1. Elevated KDR expression in myeloid cells is associated with malignant murine gliomas. (A) Representative flow cytometry graphs of CD11b and GFP in peripheral blood of KDR GFP mice bearing gliomas. Astrocytic tumors were generated by RCAS/TVA-mediated overexpression of PDGF as described in Methods. Low-grade gliomas were observed by week 5 and high grade by weeks 7-8. Controls (CTL) were mice bearing an intact Kdr locus without GFP knockin. (B) Quantification of CD11b+KDR ${ }^{\mathrm{CFP+}+}$ cell frequency in peripheral blood of RCAS/TVA tumor mice at low-grade and high-grade stages. ${ }^{* * *} P<0.001$, Student's $t$ test. LEU, Leukocytes. (C) Further characterization of murine CD11b+KDR ${ }^{\mathrm{CFP}+}$ cells in peripheral blood by Ly6C and Ly6C staining. (D) Quantification of Ly6C+ and

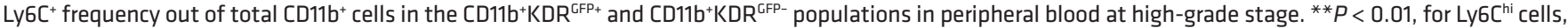

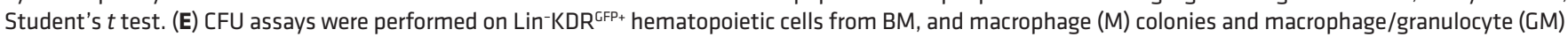
colonies, but not granulocyte (C) colonies, were observed. Quantification of various colony types formed from lineage-negative KDR ${ }^{\mathrm{CFP}-}$ or KDR ${ }^{\mathrm{CFP}+}$ cells. ${ }^{* * *} P<0.001$, in granulocytes, Student's $t$ test. (F) Gene expression (quantitative RT-PCR) of various markers, including Kdr in in vitro-cultured BM Lin ${ }^{-}$cells exposed to GL261-conditioned medium at different time points. ${ }^{*} P<0.05 ;{ }^{* *} P<0.01 ;{ }^{* *} P<0.001,1$-way ANOVA. Data are shown as mean $\pm \mathrm{SD}$.

within various models of tumor malignancy $(2,16-18)$. Recently, KDR was found to be expressed by BM-derived plasmacytoid dendritic cells (19), suggesting it may have a broader impact on tumor microenvironment than previously thought. To study the expression pattern of KDR in gliomas at different stages, we utilized $K d r^{G F P}$ mice, with GFP knocked into the $K d r$ locus, crossbred with Nestin-tva Ink4a/Arf/- Pten ${ }^{A / / l}$ mice. The replication-competent avian sarcoma-leukosis virus (ASLV) long terminal repeat (LTR) with a splice acceptor/tumor virus A (RCAS/TVA) system was used to induce PDGF/protein kinase B-driven (PDGF/Akt-driven) gliomas from neural progenitor cells in postnatal mice $(20,21)$. These mice develop low-grade gliomas that invariably progress to higher grades over a reproducible 12-week time course. Initially, at approximately week 5 , the tumors mimic fibrillary astrocytomas, which are equivalent to the low-grade human grade II tumors but subsequently progress to anaplastic astrocytomas (grade III) and, by weeks 8-12, progress to malignant gliomas akin to human glioblastoma or WHO grade IV tumors. Use of the RCAS/TVA model allowed monitoring of KDR expression throughout both the formation and malignant progression of gliomas. During tumor progression from a lowgrade stage ( week 5$)$ to a high-grade stage ( week 8$)$, the population of $\mathrm{CD}_{11} \mathrm{~b}^{+} \mathrm{KDR}^{\mathrm{GPP}+}$ cells within the peripheral blood increased 5 -fold (Figure 1, A and B). During this transition, tumor-associated macrophages within tumor tissue were also increased 5 -fold (Supplemental Figure 1A; supplemental material available online with this article; https://doi.org/10.1172/JCI86443DS1), mirroring the elevated populations of MDSCs (Supplemental Figure 1B). Within the $\mathrm{CD} 11 \mathrm{~b}^{+} \mathrm{KDR}^{\mathrm{GFP}+}$ population in peripheral blood, the majority of cells phenotypically resemble MDSCs $\left(\mathrm{Ly}_{6} \mathrm{G}^{+}\right.$or $\left.\mathrm{Ly} 6 \mathrm{C}^{+}\right)$. Approximately $60 \%$ of $\mathrm{CD}^{11} \mathrm{~b}^{+} \mathrm{KDR}^{\mathrm{GFP}+}$ cells had monocytic features versus $15 \%$ of the CD11b ${ }^{+} \mathrm{KDR}^{\text {GFP- }}$ cells (Ly6 $\mathrm{C}^{+}$; Figure $1, \mathrm{C}$ and D). Among BM progenitor cells, more than $80 \%$ of $\mathrm{Lin}^{-} \mathrm{KDR}^{\mathrm{GPP}+}$ cells

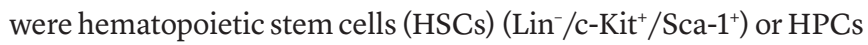
$\left(\mathrm{Lin}^{-} / \mathrm{c}-\mathrm{Kit}^{+} / \mathrm{Sca}-1^{-}\right)$(Supplemental Figure 1, C and D). 
A
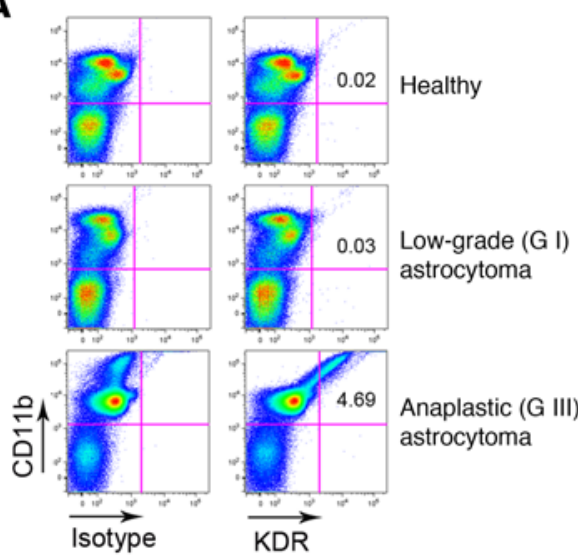

C

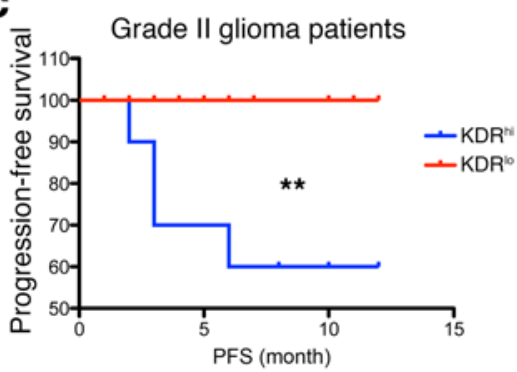

D
B
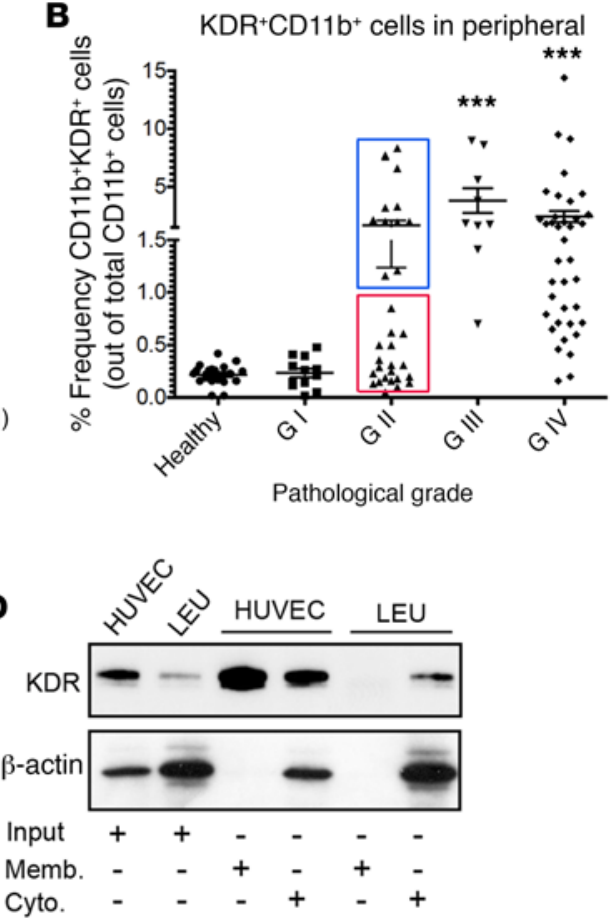

Figure 2. Elevated KDR expression is evident in myeloid cells of high-grade glioma patients. (A) Representative flow cytometry graphs of CD11b and intracellular KDR and isotype control on leukocytes of healthy subjects and patients with low-grade anaplastic astrocytoma. (B) Dot plot of CD11b+KDR+ cell frequency out of total CD11b cells in patients with different stages of glioma. Healthy donors served as controls. Grade III/grade IV versus healthy. ${ }^{* * *} P<0.001$, 1-way ANOVA. $n=111$. G1, grade I. (C) Grade II glioma patients were subdivided into 2 groups, indicated by the red and blue boxes, based on the frequency of CD11b+KDR ${ }^{+}$cells: KDR ${ }^{\text {hi }}$ and $K D R^{10}$ (cut-off, 1.0\%). Kaplan-Meier curves for progression-free survival over 12 months are presented $(n=27)$. ${ }^{*} P<0.01$, log-rank test. (D) Immunoblotting of KDR and $\beta$-actin on different fractions (cytosol [Cyto.], membrane [Memb.], input) of leukocytes (LEU) from glioblastoma patients. The experiment was conducted 3 times.
To further validate that KDR-expressing hematopoietic cells are restricted to the myeloid lineage, we isolated $\mathrm{KDR}^{\mathrm{GFP}+\text { Lin- }}$ hematopoietic cells and performed a colony formation assay, which demonstrated that these cells were capable of forming myeloid-derived colonies. Compared with lineage-negative

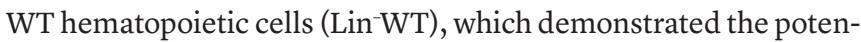
tial to form all granulocyte-macrophage, granulocyte, and macrophage colonies, $\mathrm{KDR}^{\mathrm{GFP}+/ \mathrm{Lin}-}$ cells were rarely found to potentiate granulocytic colonies (Figure 1E).

To investigate the expression pattern of KDR throughout the process of myeloid differentiation, Lin $^{-}$hematopoietic cells were grown in tumor cell line-conditioned (GL261) medium for 1 week. Compared with values on day 0 , the undifferentiated stem cell marker c-Kit was downregulated beginning on day 1 of differentiation, whereas differentiated cell marker CD11b and late-stage differentiation marker CD68 were upregulated (Figure 1F). KDR expression was maximal on day 1 and day 2 in the early differentiation phase of myeloid lineage cells; however, expression began to drop thereafter, suggesting a role for KDR in the early programming of myeloid progenitors toward a protumoral lineage.

Myeloid KDR expression was also assessed in glioma patients. As part of this study, peripheral blood samples were prospectively collected for 3 years from a cohort of glioma patients diagnosed with different tumor grades. We detected significant amounts of KDR within permeabilized leukocytes of high-grade glioma patients. As shown in Figure 2A, there was a defined CD11b ${ }^{+}$population expressing KDR. Within each sample, the ratio of $\mathrm{CD} 11 \mathrm{~b}^{+}$ $\mathrm{KDR}^{+}$versus $\mathrm{CD}_{11 \mathrm{~b}^{+}}$myeloid cells was quantified and plotted within each of the WHO-recognized pathological grades. The percentage of $\mathrm{KDR}^{+}$myeloid cells was significantly higher in blood samples derived from patients with high-grade gliomas as com- pared with samples from low-grade glioma patients (Figure 2, A

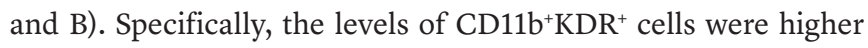
in patients with grade III or IV tumors than they were in patients with grade I tumors and healthy controls (Figure 2B). Among all patients, 2 grade III patients and 4 grade IV patients had received chemotherapy, 7 grade IV patients had received steroids, and none had received radiotherapy or targeted therapy by the time of sample collection. Importantly, the elevated levels of $\mathrm{CD}_{11} \mathrm{~b}^{+} \mathrm{KDR}^{+}$ cells were not induced by treatments that patients had received (Supplemental Figure 2, A and B).

Perhaps the most striking of our findings was the apparent delineation of 2 distinct populations of patients, each with pathologically defined grade II astrocytoma. We divided patients with grade II astrocytoma into 2 subgroups, those with high versus those

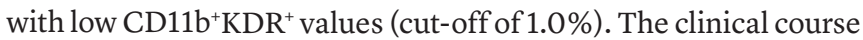
and medical histories of the patients were closely followed over 12 months. Disease progression in patients with grade II glioma was assessed by MRI and subsequent histological diagnoses of grade III or IV glioma (Supplemental Figure 2C). The progression-free survival of our cohort of grade II patients is shown in Figure 2C. Patients with an elevated percentage of $\mathrm{CD} 11 \mathrm{~b}^{+} \mathrm{KDR}^{+}\left(\mathrm{KDR}^{\mathrm{Hi}}\right)$ myeloid cells presented with a significantly higher likelihood of tumor progression from fibrillary to anaplastic variants (Figure 2C). This recognition of a subclass within grade II astrocytomas represents what we believe is a novel finding as well as a prospective paradigm shift from observation to earlier active therapy in the treatment of patients with low-grade gliomas.

Complex interactions between the host immune system and malignant tumors are an essential component of how the tumor microenvironment is thought to modulate oncologic growth and transformation $(1,11)$. Our findings suggest that high-grade glio- 
A

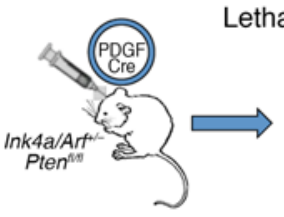

P0-P2

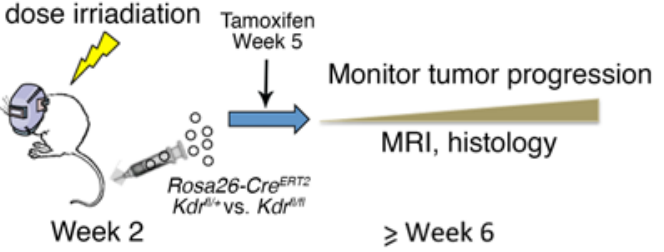

$\geqslant$ Week 6
B

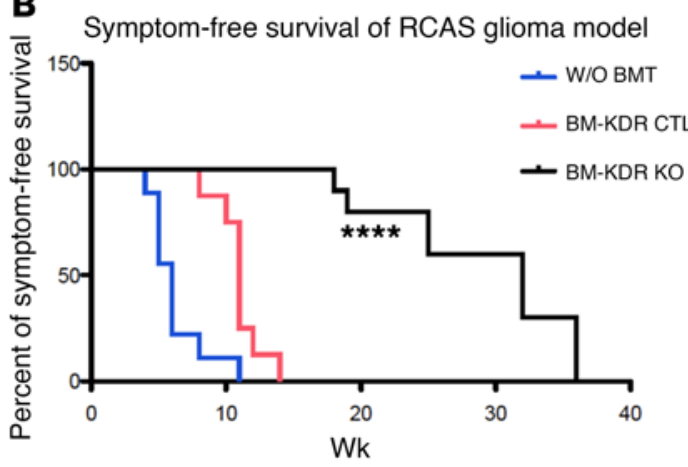

c

Week 6
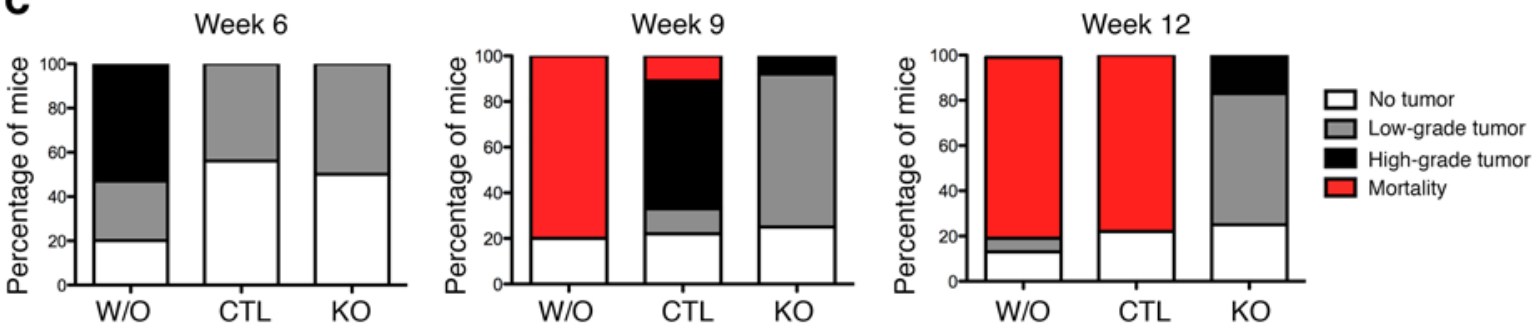

D
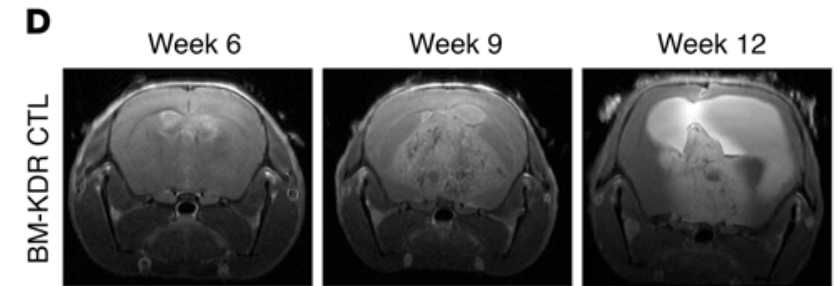

E

BM-KDR CTL

BM-KDR KO
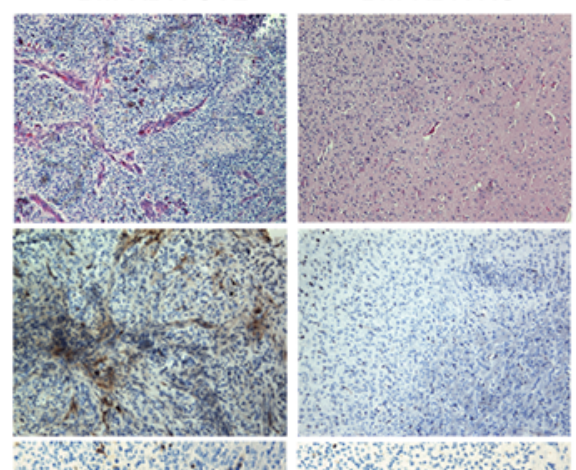

$H \& E$
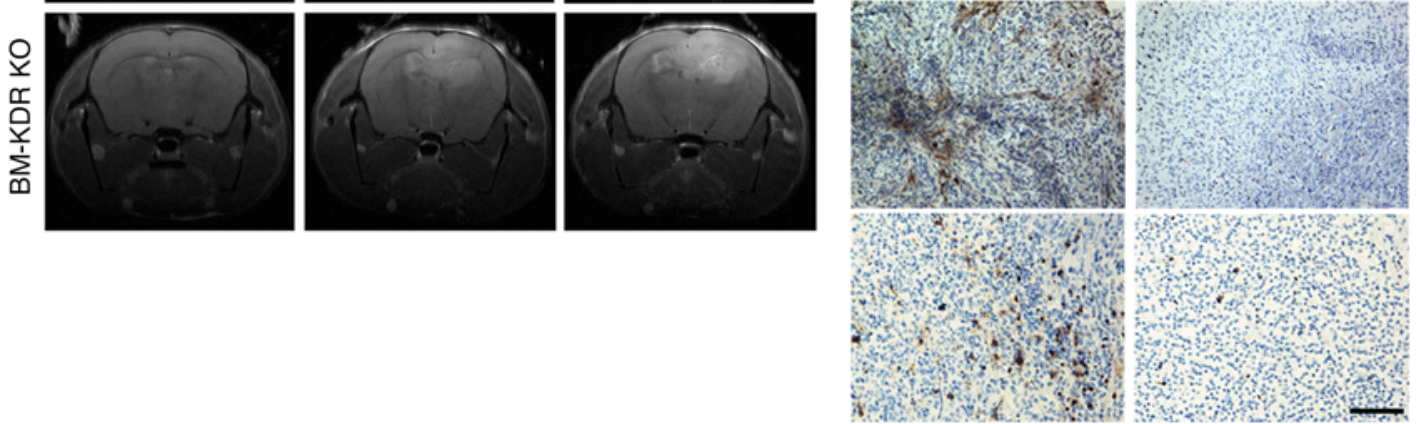

CD11b

Figure 3. KO of KDR in BMDCs suppresses spontaneous malignant transformation of RCAS/TVA gliomas. (A) Schematic diagram of experimental design. PDGF and Cre genes were transduced to PO-P2 mouse pups on an Ink4a/Arf ${ }^{\prime-}$ Pten $^{\text {fl/fl }}$ background. Mice received a lethal dose of irradiation and received bone marrow transplant (BMT) at week 2. Tamoxifen was applied to mice at approximately week 5 to induce the ablation of the KDR gene in BMDCs. Tumors were monitored by MRI and histological analyses over the process. (B) Kaplan-Meier symptom-free survival curve for RCAS/TVA mice transplanted with Rosa26-Cre ${ }^{E R T 2} K d r^{f / f f}$ and Rosa26-Cre ${ }^{E R T 2} K d r^{f / /+}$ BM cells (BM-KDR KO and BM-KDR CTL, respectively) and RCAS/TVA mice without irradiation/transplantation (without BMT). ${ }^{* * *} P<0.0001$, log-rank test. (C) At weeks 6, 9, and 12, tumors in each group of mice were assessed and graded based on MRI and further confirmation with histology. $n=19-26$ for each group. (D) Representative T2-MRI images of intracranial tumors in RCAS/TVA mice transplanted with Rosa26-Cre ${ }^{E R T 2} K d r^{f l / f l}$ and Rosa26-Cre ${ }^{E R T 2} K d r^{f / /+}$ BM cells at indicated time points. $n=12$. (E) Representative images of H\&E and IHC (CD11b, CD31) staining of tumor tissue sections from BM-KDR KO and BM-KDR control groups, respectively, at week 9. $n=10$. Scale bar: $50 \mu \mathrm{m}$.

ma patients harbor greater numbers of infiltrating myeloid cells within the tumor tissue and greater numbers of MDSC-like cells in the blood than patients with more benign phenotypes (Supplemental Figure 2, D and E). In contrast to the typical cell surface distribution of KDR in endothelial cells, our results show that KDR resides primarily within the cytoplasm of leukocytes (Supplemental Figure 3, A and B). KDR expression was not detected at significant levels in nonpermeabilized leukocytes, indicating that KDR is mainly intracellular in its localization (Supplemental Figure 3C).
The distribution of KDR on endothelial cells was also shown on HUVECs and high-grade murine glioma tissue (Supplemental Figure 3, D and E). Immunoblotting of KDR on different cellular fractions of both myeloid and endothelial cells revealed that myeloid-derived KDR was intracellular and intact (Figure 2D).

KDR controls differentiation of protumoral myeloid cells. The observation that KDR expression was temporally associated with differentiation of tumor-associated myeloid cells prompted us to closely examine the role of BM-derived KDR in the immunomodulation 

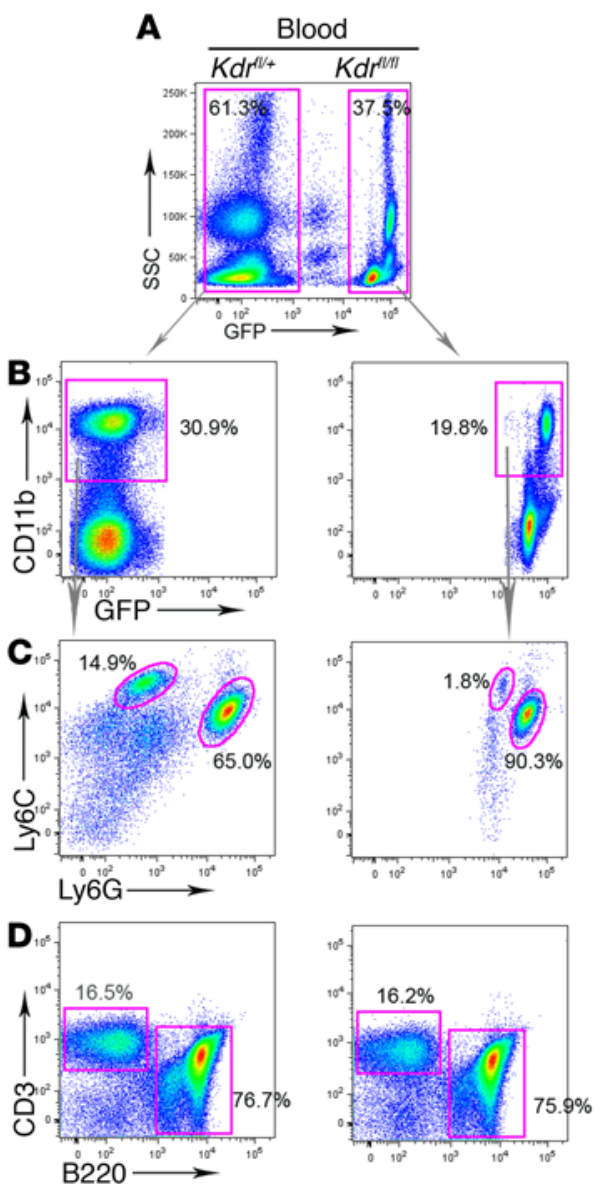

E Bone marrow
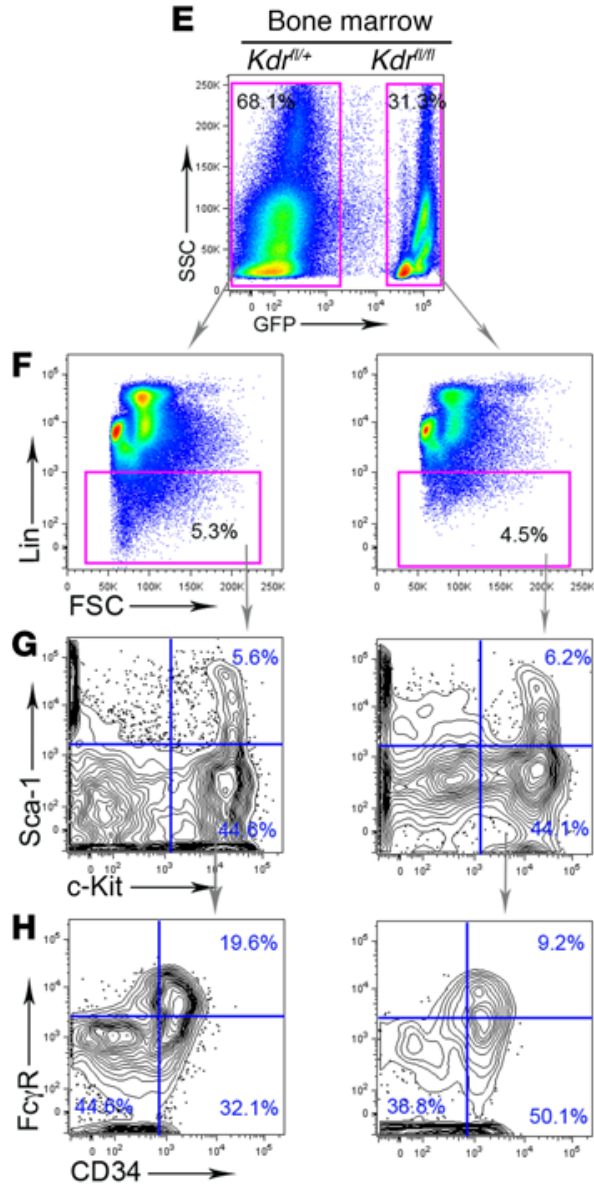

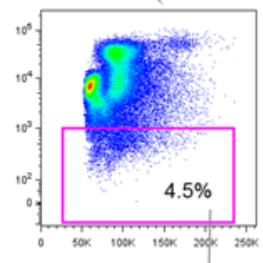

y
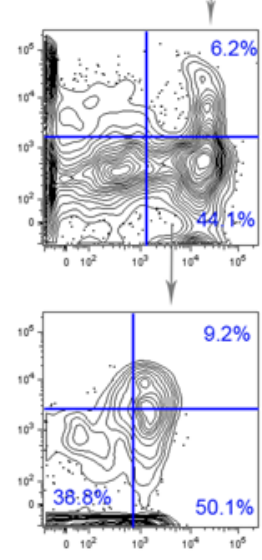

Figure 4. Competitive BMT showed that KDR KO in BM cells led to deficiency of the differentiation of monocytic cells. Lethal dose-irradiated C57BL/6 mice were transplanted with Ubc-GFP Rosa26-Cre ERT2 $^{\text {ERT }}$ $K d r^{f l / f l}$ and Rosa26-Cre ${ }^{E R T 2} K D R^{f / /+}$ BM cells, and CL261 tumors were implanted after BM engraftment. (A) Peripheral white blood cells were analyzed on side scatter (SSC) and GFP by flow cytometry. The $\mathrm{GFP}^{+}$and GFP- populations were gated for further analysis. CD11b versus GFP (B), Ly6C versus Ly6C (C), and CD3 versus B220 (D) are shown on both GFP' and GFP- populations. (E) BM cells were also analyzed on side scatter and GFP. (F) Lineage-negative cells were gated for analysis of HSCs and HPCs by c-Kit versus Sca-1 (C). (H) HPCs ( Lin $\left.^{-} \mathrm{C}-\mathrm{Kit}^{+} \mathrm{Sca}-\mathrm{1}^{-}\right)$were further characterized by Fc $\gamma R$ versus $C D 34$ within both $\mathrm{GFP}^{+}$and GFP- populations. The experiment was conducted 5 times.

of glioma progression. We therefore created a syngeneic astrocytic tumor model by orthotopic injection of GL261 cells into the frontal lobes of C57BL/6 mice. These mice harbored BM that had been

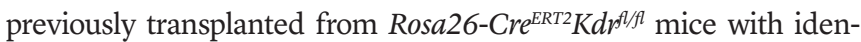
tical C57BL/6 backgrounds. The efficiency of KDR depletion and the subsequent effects upon hematopoiesis within this system were then assessed. The KDR allele was deleted in more than $80 \%$ of $\mathrm{BM}$ cells, and a complete blood count was not significantly affected by knocking out KDR (Supplemental Figure 4, A-D). At day 3 after tumor implantation, tamoxifen was administered to suppress the expression of KDR within BMDCs. Tumor growth was monitored via daily bioluminescence and weekly MRI scans. Tumor growth was significantly suppressed, by approximately $25 \%(P<0.05)$ (Supplemental Figure $5 \mathrm{~A}$ ), by selectively knocking out $K D R$ within BMDCs $\left(\mathrm{BM}-\mathrm{KDR} \mathrm{KO}, K D R^{f / f}\right)$ when compared with controls (BM-KDR $\left.\mathrm{CTL}, K D R^{f /+}\right)$. We next assayed for the presence of myeloid cells (CD11b) and blood vessels (collagen IV) in GL261 tumors in KDR $R^{f / f l}$ mice. These animals had a marked absence of tumor-associated myeloid cells within both the tumor core and the tumor periphery (Supplemental Figure 5B). While bioluminescence demonstrated only subtle differences in tumor burden between the 2 groups, MRI revealed a striking difference, particularly in the sequences measuring glioma contrast enhancement. This result suggested a clear and direct effect of KDR deficiency within BMDCs upon tumor vascularity. GL261 tumors in the control $\left(K D R^{f /+}\right)$ group demonstrated significantly more enhancement when compared with animals with KDR-depleted BM $\left(K D R^{f / f l}\right)$, as measured by gadolinium- diethylenetriamine penta-acetic acid (gadolinium-DTPA) and gadolinium-albumin, which closely approximates blood flow (Supplemental Figure 6, A and B). Quantification of MRI on day 18 showed that brain tumors in the $K D R^{f / f l}$ animals were $25 \%$ smaller and showed an $80 \%$ decrease in their contrast enhancement when compared with tumors in control animals (Supplemental Figure 6, C and D).

Endothelial and pericyte staining within tumors grown in $K D R^{f / f l}$ mice demonstrated marked differences in blood vessel morphology, vasculature size, and pericyte coverage compared with those in the control group (Supplemental Figure 7, A and B). Wide-field tumor histology in tumors from $K D R^{f / f l}$ mice demonstrated less hemorrhage at the tumor core and a less invasive tumor margin when compared with those in $K D R^{f l+}$ mice (Supplemental Figure $7 C$ ).

We noted that the microenvironment of xenograft brain tumor models may not always accurately reflect the complexity of invasive de novo brain tumors and thus approximated this intricacy more accurately by challenging findings from the GL261 allograft system using the spontaneous RCAS/TVA murine brain tumor model (20). In order to examine the role of KDR in BMDCs from RCAS/TVA glioma mice, we irradiated and transplanted RCAS/TVA mice at week 2 with Rosa26-Cre $e^{E R T 2} K d r^{f l / f l}$ or Rosa26-Cre $e^{E R T 2} K d r^{f /+}$ BMDCs from littermate donor mice. Tamoxifen was administered to induce targeted deletion of the KDR locus specifically within BMDCs before host animals first showed evidence of astrocytic tumors at approximately week 5. Beginning at week 6, recipient RCAS/TVA mice were monitored for tumor progression using weekly MRI scans and histological samples from representative sacrificed mice 
A
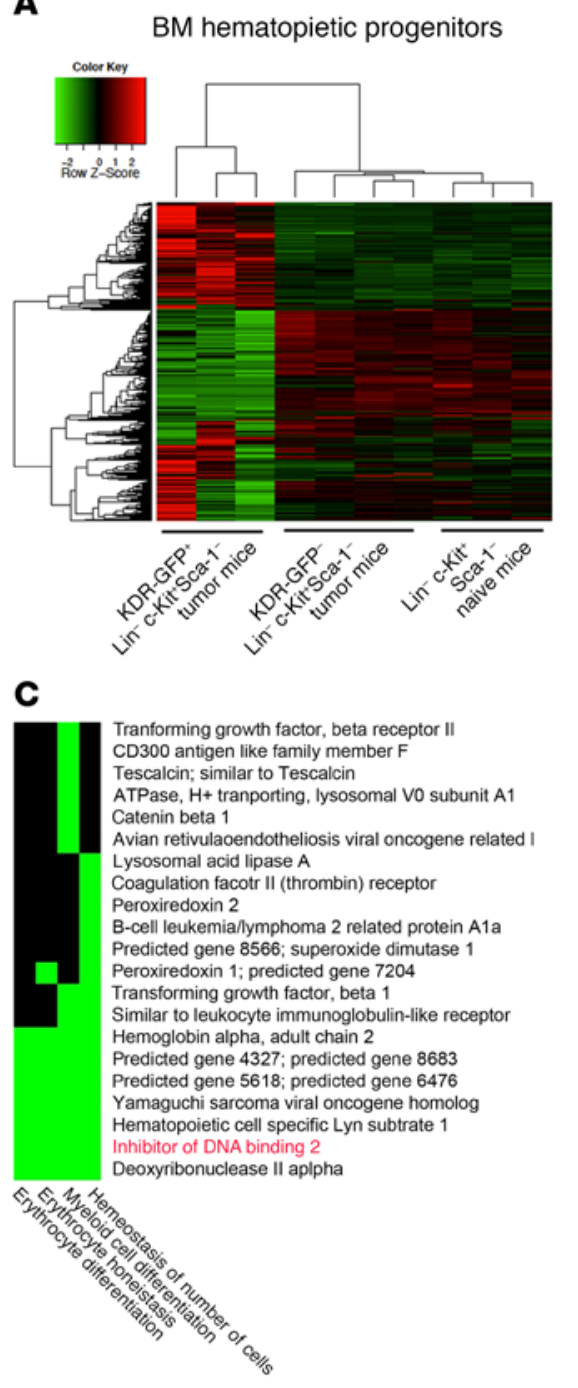

E

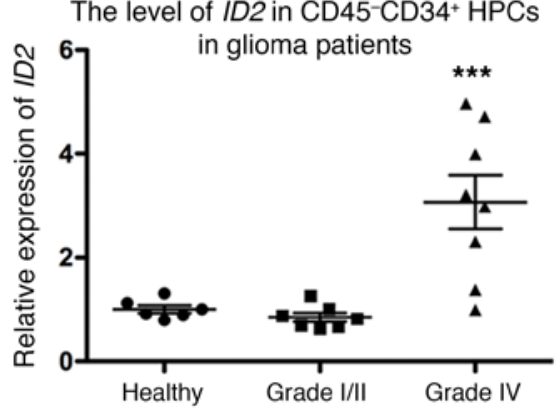

B
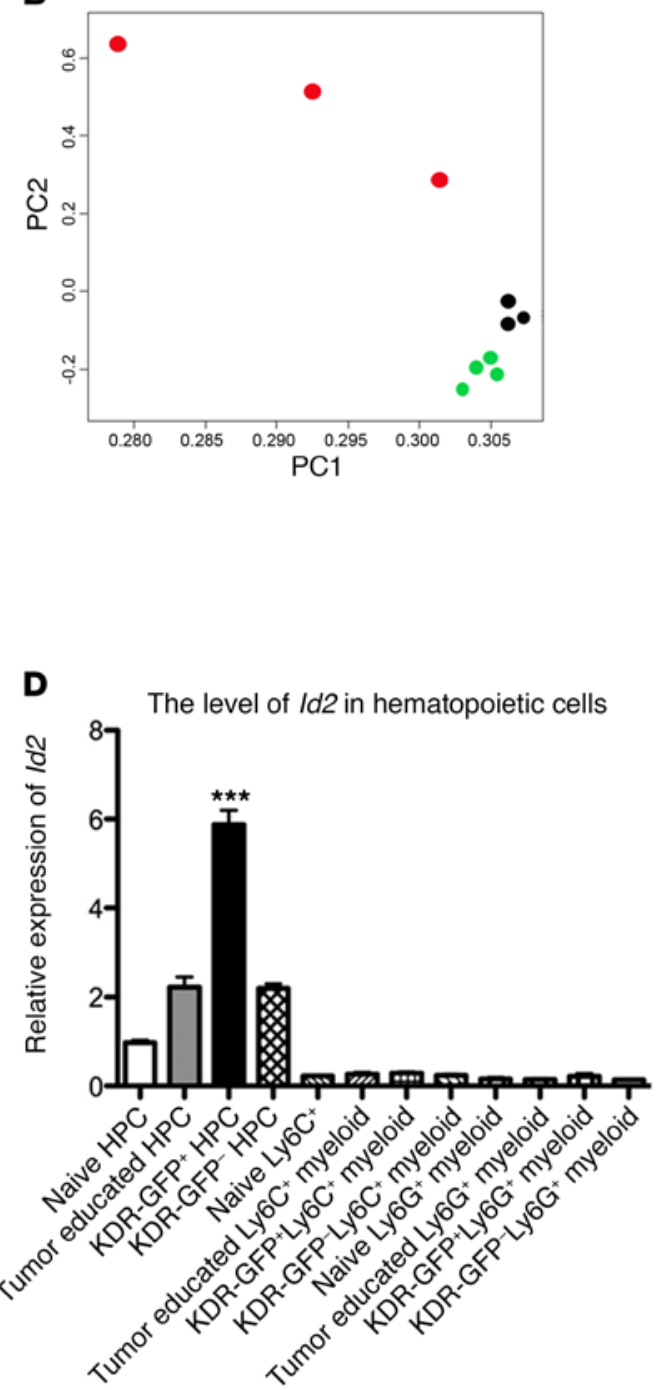

$\mathbf{F}$

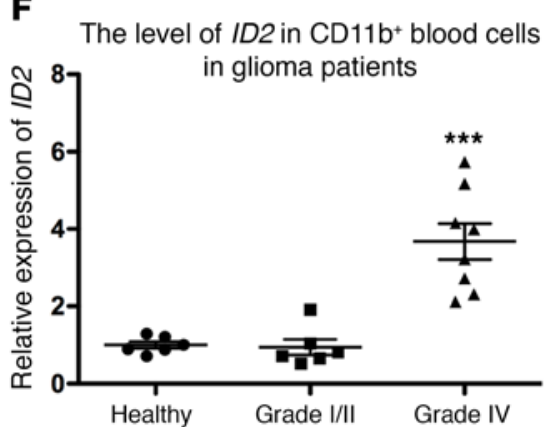

Figure 5. RNA-sequencing reveals gene profiles for KDR ${ }^{+}$BM cells. (A) The unsupervised principal component analysis of significantly altered genes in $\mathrm{Lin}^{-} \mathrm{c}-\mathrm{Kit}^{+}$ Sca-1- from naive mice, Lin ${ }^{-}{ }^{-}-\mathrm{Kit}^{+} \mathrm{Sca}-{ }^{-1}$ KDR-GFP+ cells, and Lin ${ }^{-}-\mathrm{Kit}^{+}$Sca-1-KDRGFP- cells from BM of tumor-bearing mice. (B) Lin'c-Kit ${ }^{+} S c a-1^{-}$cells from naive mice (black), Lin'c-Kit ${ }^{+} S c a-1^{-}$KDR-GFP+ cells (red), and Lin'c-Kit ${ }^{+}$Sca-1-KDR-GFP- cells (green) from BM of tumor-bearing mice, based on similarity of gene profiles. PC1, principal component 1. (C) The candidate genes $(P<0.05,>1.5$-fold change; Lin'c$\mathrm{Kit}^{+} \mathrm{Sca}-\mathrm{1}^{-}$KDR-GFP+ versus Lin ${ }^{-} \mathrm{C}^{-\mathrm{Kit}^{+} \mathrm{Sca}-}$ 1-KDR-GFP-) were divided according to the subset with the highest expression and analyzed for categories with significant enrichment $(P<0.05)$ of categories in GO biologic processes using DAVID tools. Similar categories were grouped accordingly. The presence of association between functions and genes was color highlighted (black [negative] versus green [positive]).

(D) Expression of $I d 2$ in various lineages of hematopoietic cells. ${ }^{* *} P<0.001$, 1-way ANOVA. (E) Expression of ID2 in HPCs $\left(\mathrm{CD}_{45}{ }^{-} \mathrm{CD} 34^{+}\right)$from patients with low-grade or high-grade gliomas. ${ }^{* *} P<0.001$, 1-way ANOVA. $n=21$. (F) Expression of $I D 2$ in CD11b+ blood cells from patients with lowgrade or high-grade gliomas. ${ }^{* *} P<0.001$, 1 -way ANOVA. $n=20$. Data are shown as mean \pm SD. were analyzed to confirm anaplasia or malignant transformation when scans were suggestive of transformation (Figure 3A). Three cohorts of RCAS/TVA mice (i.e., those that received Rosa26$C r e^{E R T 2} K d r^{f / f l}$ or control Rosa26-Cre ${ }^{E R T 2} \mathrm{Kdr} r^{f /+} \mathrm{BM}$ and those that did not undergo irradiation-transplantation) were analyzed based on their symptoms associated with late-stage glioma. These symptoms included hydrocephalus, lethargy, and weight loss. Kaplan-Meier symptom-free survival curves were utilized to demonstrate glioma progression in the 3 cohorts. Median symptom-free survival time (MSST) was 11 weeks for RCAS/TVA mice transplanted with control Rosa26-Cre ${ }^{E R T 2} K d r^{f /+} \mathrm{BM}$ versus 32 weeks for RCAS/TVA mice transplanted with Rosa26-Cre ${ }^{E R T 2} K d r^{f / f l}$ BM. For RCAS/TVA mice not treated with irradiation or a bone marrow transplant (BMT), MSST was 6 weeks (Figure 3B). We compared brain tumors in RCAS/TVA mice at weeks 6, 9, and 12 using MRI followed by histological examination (Figure $3 \mathrm{C}$ ). Knocking out $\mathrm{Kdr}$ in BMDCs 
A
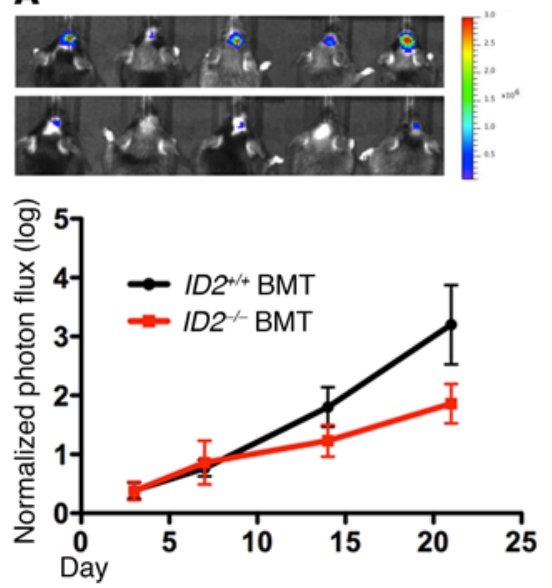

C

B
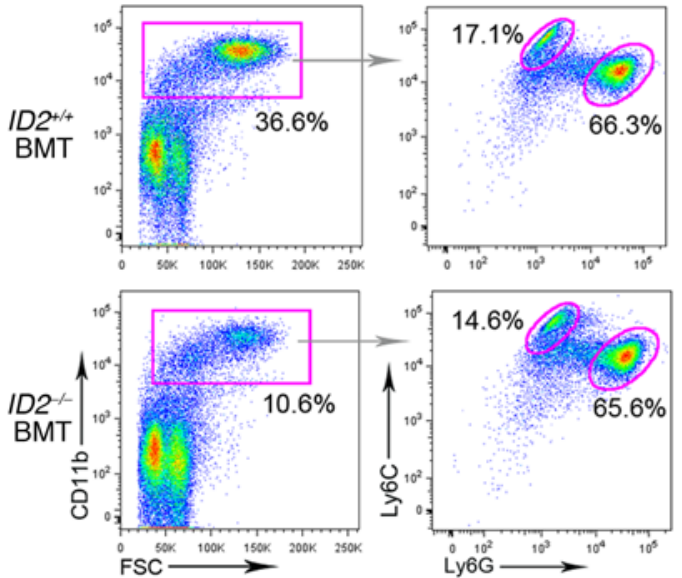

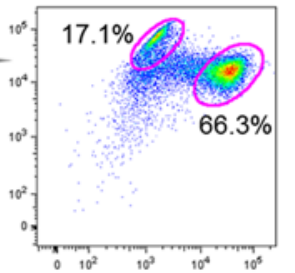

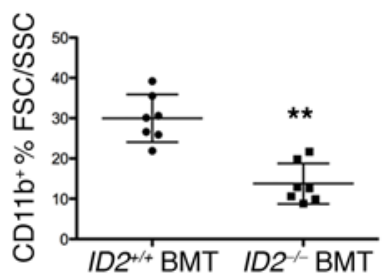

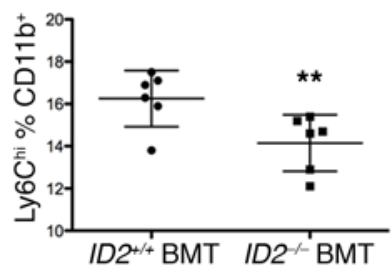

ID2+/+ $\mathrm{BMT} \quad I D 2^{-1-} \mathrm{BMT}$

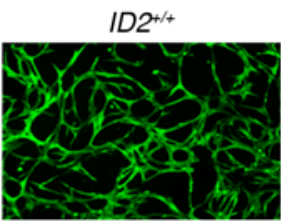

D
$I D 2^{+/ 4}$
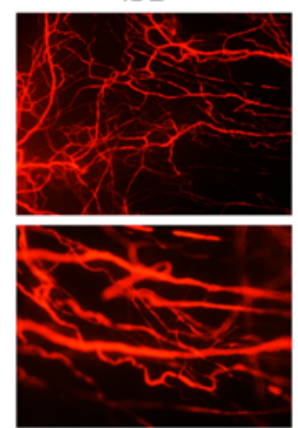
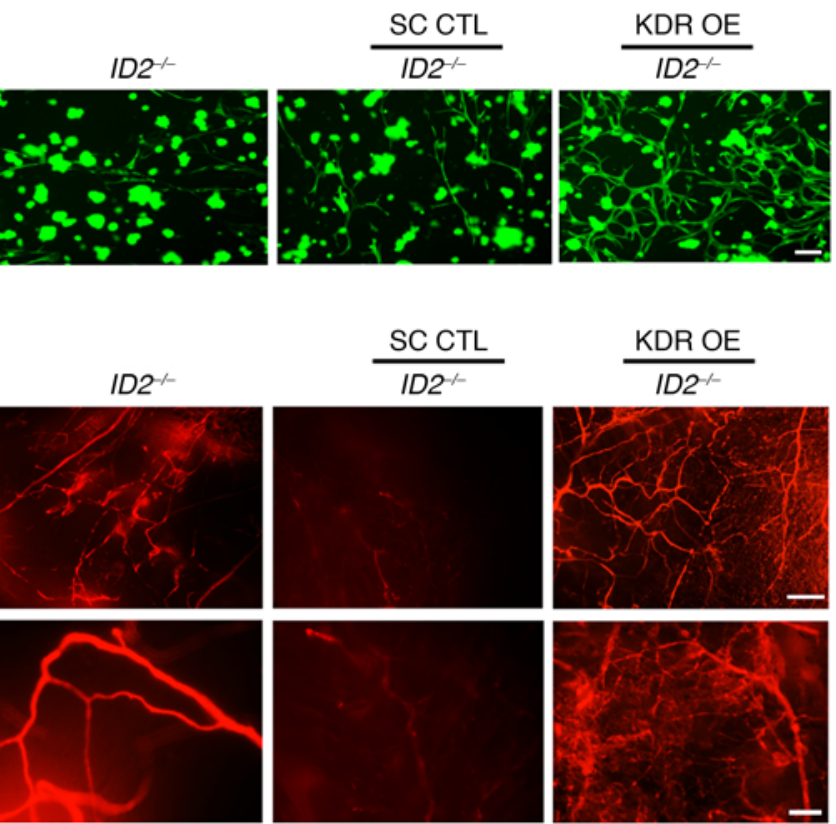
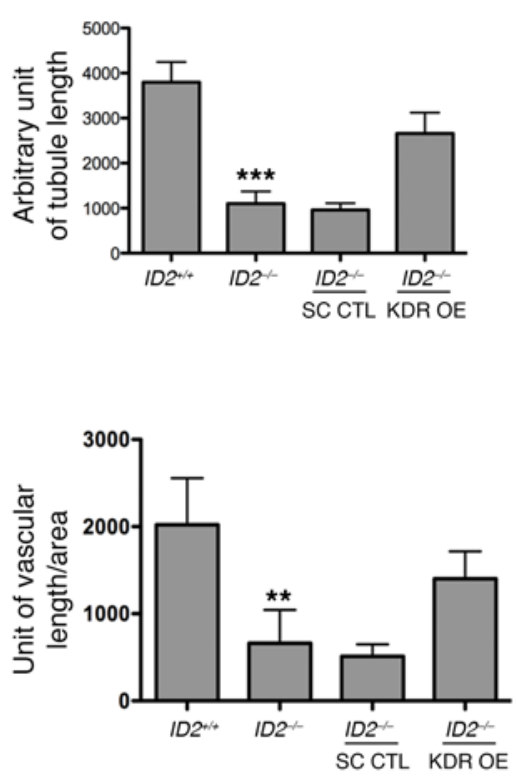

Figure 6. ID2 promotes proangiogenic phenotypes of tumor-associated myeloid cells. (A) Chimeric C57BL/6 mice transplanted with Id2 $2^{-/-}$BM cells (Id $2^{+/+}$ BM cells as control) were implanted with luciferase-labeled GL261 tumors intracranially. Tamoxifen was applied at day 3 after implantation. The tumors were monitored by bioluminescence. Representative images were taken at day 14 . The color bar on the right represents photon intensity. The experiments had 2 replicates. The tumor growth curve is based on bioluminescence. $n=10 .{ }^{*} P<0.01,1$-way ANOVA. (B) Flow cytometry analysis of peripheral blood cells on CD11b, Ly6C, and Ly6C in Id2 $2^{+/}$BMT and $/ d 2^{-/-}$BMT groups. Quantification of CD11 b+ cell frequency out of total white blood cells and of Ly6C ${ }^{\text {hi }}$ cell frequency out of CD11b+cells. ${ }^{*} P<0.01$, 1-way ANOVA, for each group. $n=7$. (C) Tubule formations of HCMEC/D3 (GFP) cocultured with lineage-negative

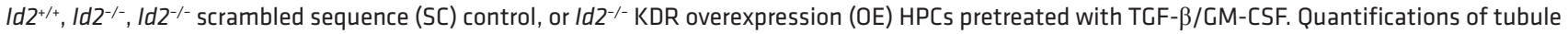

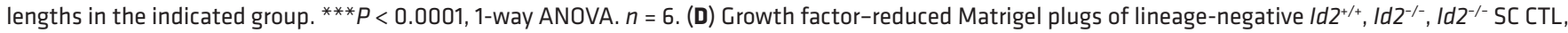
or Id $2^{-/-}$KDR overexpression HPCs pretreated with TCF- $\beta /$ GM-CSF. Blood vessels (red) were perfused with rhodamine-dextran. Lower panels show magnified views to highlight vascular permeability. Quantifications of based blood vessels density (BVD) in each group. ${ }^{* *} P<0.01,1$-way ANOVA. $n=6$. Data are represented as mean \pm SD. Scale bars: $50 \mu \mathrm{m}$ (D, upper panels); $20 \mu \mathrm{m}$ (C, D, lower panels).

substantially delayed the malignant transformation of low-grade gliomas to higher grade states. By week $9,60 \%$ of RCAS/TVA mice transplanted with control Rosa26-Cre ${ }^{E R T 2} \mathrm{Kdr} r^{f /+} \mathrm{BM}$ developed highgrade tumors that had typical malignant features, including robust gadolinium enhancement on MRI, pseudopalisading necrosis, and microvascular proliferation (Figure 3, D and E). In contrast, tumors in $70 \%$ of RCAS/TVA mice transplanted with Rosa26-Cre ${ }^{E R T 2} \mathrm{Kdrt/fl}$ $\mathrm{BM}$ remained in the low-grade stage without signs of malignant transformation at week 9 (Figure 3, C-E).
ID2 mediates KDR expression and granulocytic/monocytic cell differentiation. Our data demonstrate that KDR contributes to the differentiation of proangiogenic myeloid cells and, more importantly, suggest that these cells play a major role in the modulation of gliomas. Within this context, we sought to examine the mechanistic and functional role KDR has in directing the hematopoietic fate of KDR-expressing BMDCs in protumorigenic immune cells.

We began by analyzing myeloid cell differentiation either in the presence or absence of KDR throughout distinct phases of tumor 
progression. BM cells harvested from $\mathrm{GFP}^{+}$Rosa26-Cre ${ }^{E R T 2} \mathrm{Kdr} r^{\mathrm{t} / \mathrm{fl}}$ and $\mathrm{GFP}^{-}$Rosa26-Cre ${ }^{E R T 2} \mathrm{Kdr} \mathrm{r}^{\mathrm{l}+\mathrm{+}}$ mice were mixed in a 1:1 ratio and transplanted into recipient mice. These mice were subsequently implanted with orthotopic GL261 tumors. Populations of myeloid cells, T cells, and B cells residing in the peripheral blood are shown in Figure 4, A-D. Populations of HSCs and myeloid progenitors residing in $\mathrm{BM}$ are shown in Figure 4, $\mathrm{E}-\mathrm{H}$. After $\mathrm{BM}$ engraftment, $\mathrm{GFP}^{+}$Rosa26-Cre ${ }^{E R T 2} \mathrm{Kdrf/fl} \mathrm{BM}$ cells reconstituted $35 \%-40 \%$ of hematopoietic cells in both the BM and peripheral blood, whereas the remainder contained $\mathrm{GFP}^{-}$Rosa26-Cre ${ }^{E R T 2} \mathrm{Kdr} r^{f /+} \mathrm{BM}$ cells as well as the remaining host hematopoietic cells not eliminated by the radiation protocol (Figure 4, A and E). Compared with the control $\mathrm{GFP}^{-}$population in peripheral blood, the $\mathrm{GFP}^{+}$population had fewer total CD11b+ cells (Figure 4B and Supplemental Figure 8A), which contained fewer Ly6C $\mathrm{C}^{+}$cells (Figure $4 \mathrm{C}$ and Supplemental Figure $8 \mathrm{~B})$. There was no significant difference in $\mathrm{T}$ cell $\left(\mathrm{CD}^{+}\right)$and $\mathrm{B}$ cell $\left(\mathrm{B} 22 \mathrm{O}^{+}\right.$) lineages between $\mathrm{GFP}^{+}$and $\mathrm{GFP}^{-}$populations (Supplemental Figure 8, G and $\mathrm{H}$ ). Within the $\mathrm{BM}$, the $\mathrm{GFP}^{+}$population had levels of lineage-negative cells and HSCs $\left(\mathrm{Lin}^{-} \mathrm{c}-\mathrm{Kit}^{+} \mathrm{Sca} 1^{+}\right)$that were comparable to those in the $\mathrm{GFP}^{-}$population (Figure $4, \mathrm{~F}$ and G, and Supplemental Figure 8, C and D). Interestingly, within HPCs ( Lin $^{-} \mathrm{c}-\mathrm{Kit}^{+} \mathrm{Sca1}{ }^{-}$), the $\mathrm{GFP}^{+}$population had significantly more common myeloid progenitor cells ( $\left.\mathrm{Lin}^{-}{ }^{-}-\mathrm{Kit}^{+} \mathrm{Sca}{ }^{-} \mathrm{Fc} \gamma \mathrm{R}^{-} \mathrm{CD} 34^{+}\right)$and significantly fewer granulocytic/monocytic myeloid progenitor cells $\left(\mathrm{Lin}^{-} \mathrm{c}-\mathrm{Kit}^{+} \mathrm{Sca1}{ }^{-} \mathrm{Fc} \gamma \mathrm{R}^{+} \mathrm{CD} 34^{+}\right)$in comparison with the $\mathrm{GFP}^{-}$population (Figure $4 \mathrm{H}$ and Supplemental Figure 8, $\mathrm{E}$ and $\mathrm{F}$ ). This indicates that by selectively knocking out $K d r$ in BMDCs, the differentiation of common myeloid progenitor cells into granulocytic/monocytic myeloid progenitor cells was suppressed. We performed in vitro colony formation assays using $\mathrm{K}^{+/-}$and $\mathrm{Kdr}^{-/-} \mathrm{BM}$ cells and observed significantly fewer granulocytic macrophage and macrophage colonies forming from $\mathrm{Kdr}^{-/} \mathrm{BM}$ cells (Supplemental Figure 8I).

To further define the KDR signaling network responsible for directing differentiation down a myeloid lineage, we performed gene-expression profiling of KDR-expressing ( $\mathrm{KDR}^{\mathrm{GFP}+}$ ) $\mathrm{HPCs}$ $\left(\mathrm{Lin}^{-} \mathrm{c}-\mathrm{Kit}^{+}\right)$using mRNA sequencing. Differentially expressed genes from $\mathrm{KDR}^{\mathrm{GFP}+}$ versus $\mathrm{KDR}^{\mathrm{GFP}-} \mathrm{HPCs}$ were also clustered and displayed using a heat map (Figure 5A), and similar categories were grouped accordingly (Figure 5B). Candidate genes $(P<0.001,>2$-fold change) were divided according to subsets with the highest expression, and categories with significant enrichment $(P<0.001)$ in Gene Ontology (GO) were derived using DAVID tools (22) (Figure 5C). Id2 was identified as a markedly upregulated gene in $\mathrm{KDR}^{\mathrm{GFP}+}$ HPCs. As a well-defined transcriptional regulator controlling the timing of cell-fate determination and the differentiation of stem and progenitor cells, ID2 was a strong candidate for an upstream modulator of myeloid differentiation through KDR signaling. To validate the upregulation of $I d 2$ in HPCs, we performed real-time PCR on $\mathrm{KDR}^{\mathrm{GFP}+} \mathrm{HPCs}$ and Ly $6 \mathrm{C}^{+} / \mathrm{Ly} 6 \mathrm{G}^{+}$myeloid cells from tumor-bearing or naive mice. $\mathrm{KDR}^{\mathrm{GFP}+} \mathrm{HPCs}$ from tumor-bearing mice had higher Id2 levels than did $\mathrm{KDR}^{\text {GFP- }}$ HPCs and HPCs from naive mice (Figure 5D). The expression of $I d 2$ was generally lower in myeloid cells than in HPCs. We also examined the expression of ID 2 in glioma patients. HPCs and CD11b cells were isolated from both healthy controls and low- and high-grade glioma patients. HPCs and CD11b ${ }^{+}$cells from high-grade glioma patients had significantly higher levels of
ID2 when compared with those from healthy controls and lowgrade glioma patients $(P<0.0001$, Figure $5, \mathrm{E}$ and $\mathrm{F})$.

ID2 functions by inhibiting the binding of E proteins to DNA (23). To investigate whether ID2 interacted with $\mathrm{E}$ proteins to regulate cell fate within a myeloid lineage, we analyzed the promoter region plus the $5^{\prime}$ UTR of $K d R$ for putative E protein-binding sites, known as E boxes. We identified a likely binding site for E2A (Supplemental Figure 9A) within the upstream promoter region of the $K d r$ gene. To conclusively establish the ID2/E protein/Kdr interaction, we performed a ChIP assay using E2A antibody in $I d 2^{+/+}, I d 2$ overexpressing, and $I d 2^{-/}$BM cells. As expected, the anti-E2A antibody was able to successfully pull down the upstream fragment of $K d r$. Overexpression of ID2 abolished the binding of E2A to the promoter of the $K d r$ gene (Supplemental Figure 9B); however, the binding capacity of E2A was comparable between the $I d 2^{+/+}$and $I d 2^{-/-}$groups. We hypothesize that the basal level of ID2 in WT cells is not high enough to release E2A from DNA. Granulocyte-macrophage CSF (GM-CSF) and TGF- $\beta$ induced expression of ID2 and KDR in lineage-negative BM cells (Supplemental Figure 9C), similarly to conditioned medium derived from GL261 cells, blocking either reversed ID2 or KDR expression. These results support the hypothesis that glioma-secreted GM-CSF and TGF- $\beta$ initiate protumoral myeloid cell differentiation, directing formation of the $\mathrm{KDR}^{+}$proangiogenic immune cell population, which plays a crucial role in tumor microenvironment and malignant glioma transformation. As shown in Supplemental Figure 9D, both total KDR and activated KDR were abolished by ID2 deficiency in BMDCs.

ID2/KDR axis directs proangiogenic function of myeloid cells. MDSCs are key players in antitumor immunity and angiogenesis in several reported malignancies (24-27), and the data from our patient cohort and animal models support a central role for these cells in the transformation of gliomas. We wanted to further understand the contribution of the ID2 signaling axis in regulating the differentiation of protumoral and angiogenic myeloid cells within the context of glioma immunomodulation. To begin this analysis, C57BL/6 mice that received $I d 2^{+/+}$or $I d 2^{-/-}$BMTs were orthotopically implanted with GL261 tumor cells. GL261 astrocytic tumors grew more slowly in mice with $I d 2^{-/-} \mathrm{BM}$ compared with those with $I d 2^{+/+} \mathrm{BM}(P<$

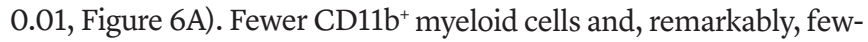
er CD $11 \mathrm{~b}^{+} \mathrm{Ly}_{6 \mathrm{C}}{ }^{+}$cells were recruited to tumors in mice with $I d 2^{-/-} \mathrm{BM}$ compared with those with $I d 2^{+/+} \mathrm{BM}(P<0.01$, Figure 6B). While the endothelial-derived VEGF/KDR signaling axis is well established within the regulation of tumor angiogenesis (28), its role in proangiogenic myeloid differentiation has not been previously established.

To determine candidate downstream targets of ID2 pathway signaling, we employed real-time PCR (RT-PCR), utilizing in vitro differentiated BMDCs from ID2-competent or ID2-incompetent tumor-bearing animals. Among target genes identified in this assay (Supplemental Figure 10A), we observed downregulation of matrix metallopeptidase $9(\mathrm{Mmp} 9)$, hypoxia inducible factor $1 \alpha$ (Hifla), and angiopoietin-2 along with $K d r$ in $I d 2^{--}$BMDCs compared with $I d 2^{+/+}$BMDCs $(P<0.01$, Supplemental Figure 10, B and C). These molecules have been previously described as essential components of the tumor microenvironment and play an important role in vascular remodeling and angiogenesis (29-31). As shown in Supplemental Figure 10D, Mmp9, Hifla, and angiopoietin-2 were upregulated in $\mathrm{CD}_{11} \mathrm{~b}^{+}$blood cells of mice with high-grade tumors compared with those with low- 
grade tumors. These proangiogenic factors are also significantly higher in $\mathrm{KDR}^{+}$myeloid cells than those in $\mathrm{KDR}^{-}$myeloid cells (Supplemental Figure 10E). To further define the proangiogenic functions of the ID2/KDR pathway within our identified myeloid cell population, we performed in vitro tube-forming assays under different conditions. We coincubated GFP-brain endothelial cells (HCMEC) with previously in vitro-differentiated $I d 2^{+/+}$and $I d 2^{-/-}$ BMDCs. In order to determine whether KDR overexpression would have a discernible effect on myeloid cells in the absence of ID2, Id2 ${ }^{--}$BMDCs were also transduced by lentivirus overexpressing either a scrambled control or the WT Kdr gene. ID2expressing BMDCs strikingly promoted tube formation; however, ID2-deficient BMDCs were unable to support the formation of endothelial tubules. KDR overexpression allowed BMDCs to regain their protubule-promoting capability $(P<0.0001$, Figure $6 C)$. In parallel, we performed an in vivo Matrigel plug assay utilizing the same BMDC conditions. $I d 2^{+/+}$BMDCs demonstrated potent induction of neovascularization in Matrigel plugs $(P<0.01$, Figure 6D), whereas loss of ID2 compromised the proangiogenic functions of BMDCs. Once again, overexpression of KDR rescued the proangiogenic phenotype of BMDCs. Interestingly, while the overexpression of $\mathrm{KDR}$ in $\mathrm{Id} 2^{--}$BMDCs showed enhanced proangiogenic capability, the blood vessels induced by $I d 2^{-/-}$BMDCs with overexpression of KDR were noticeably leakier than were those induced by WT BMDCs, as was evidenced by permeability of dextran. While we demonstrated that overexpression of KDR in BMDCs convincingly rescues the angiogenic deficiency induced by loss of the $I d 2$ gene, the histoarchitecture remains imperfect and warrants further investigation.

\section{Discussion}

KDR has been previously described as a marker for endothelial progenitor cells and has been considered a specific marker for endothelial progenitors within BMDCs $(1,18,32-34)$. Recently, however, KDR was identified in plasmacytoid dendritic cells during innate immune responses, suggesting additional roles for KDR in physiological processes (19). Using multiple complimentary approaches, we have demonstrated the expression and unique role of KDR in myeloid cells. Despite its lower expression levels in BMDCs compared with endothelial cells, KDR in BMDCs controls the differentiation of HPCs into proangiogenic myeloid cells, thereby directly affecting the composition of the tumor microenvironment. The majority of KDR-expressing BMDCs are

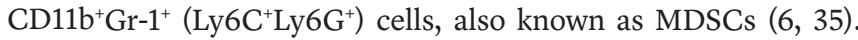
Besides the canonical immunosuppressive role of MDSCs, we observed distinct phenotypes on tumor-associated vascular development when KDR was selectively deleted from BM cell populations. Within the tumor microenvironment, macrophages and neutrophils have been shown to have robust proangiogenic capabilities in multiple disease states $(4,24,36)$. Until now, the mechanisms by which MDSCs differentiate into tumor-associated macrophages or neutrophils have not been convincingly demonstrated $(26,37)$; however, in our study, elimination of KDR-expressing BMDCs yielded fewer myeloid cells and normalized blood vessels within the tumor microenvironments. Our results suggest that KDRmediated proangiogenic myeloid cells are crucial for malignant progression in fibrillary astrocytomas.
Previous research has demonstrated that KDR can also be found in the cytosol and nucleus in addition to its known localization on the cell membrane. KDR translocates to the inside of cells upon VEGF stimulation in a process that requires phosphorylation of the receptor $(28,38,39)$. It has become clear that endocytosis and intracellular trafficking of KDR is key to the activation of VEGFR signaling (40-42). In connection with their proangiogenic functions, tumorassociated myeloid cells and myeloid progenitors serve as a major source of VEGF in many types of malignant lesions $(4,43)$. Given this information, we hypothesize that high concentrations of VEGF within myeloid cells restrain its receptor, KDR, from being localized on the cell surface. Instead, the majority of KDR remains within intracellular compartments of myeloid cells in an activated state.

Recent studies have demonstrated that malignant lesions polarize macrophages and neutrophils within the tumor microenvironment and redirect them toward tumor-promoting phenotypes $(24,44,45)$. Prior to arriving at and populating tumor tissues, BMDCs receive soluble tumor-directed signaling $(7,18,46)$. As they progress, malignancies evade immune surveillance by promoting immunosuppressive cells and suppressing antigen-presenting cell functions $(26,47)$. Primary tumors also prime BMDCs to facilitate neovascularization, allowing for exponential growth. Infiltration of several types of proangiogenic BMDCs, such as Tie-2+-expressing monocytes and $\mathrm{c}-\mathrm{Met}^{+}$hematopoietic progenitors, increases as primary tumors progress $(7,46)$. Since these proangiogenic myeloid cells appear to have a direct role within malignant transformation, they may serve as prognostic markers of early transformation in cancer patients with low-grade gliomas (48-50). For the majority of solid tumors, staging and prognosis are based upon direct tumor biopsies, which lack meaningful information about the tumor microenvironment (50). After surgery, most patients receive radiation therapy, chemotherapy, and/or targeted therapy, from which the tumor microenvironment may be affected dynamically and extensively. Considering the high incidence of tumor transformation, progression, and recurrence within patients with low-grade gliomas, it is important to consider real-time monitoring approaches in addition to MRI imaging in glioma patients.

Inhibitors of DNA-binding proteins have been shown to play important roles in mediating hematopoietic differentiation in physiological settings $(23,51)$. It has been shown, for example, that ID2 serves as a suppressor of lymphoid and B cell development $(52,53)$; however, the role of ID2 in myeloid differentiation within malignancies has not been as well described in the literature $(6,7)$. In this study, we have demonstrated that E2A, the binding partner of ID2 $(23,53)$, directly binds the $K d r$ promoter region, illuminating a crucial link between ID2 and myeloid differentiation. We have shown that enhanced ID2/KDR signaling, induced by primary glioma, promotes the differentiation of MDSCs, particularly along a monocytic lineage. These results have expanded upon the previous understanding of KDR as simply an endothelial activator and elucidated a role for KDR as a molecular regulator responsible for directing proangiogenic myeloid cell differentiation. This suggests that ID2 may play an important part in protumoral MDSC formation. Targeting the ID2/KDR axis within myeloid-derived cells may serve as an extratumoral therapeutic approach for suppressing glioma progression through immunomodulation of the tumor microenvironment. 


\section{Methods}

Cell lines and mouse lines. C57BL/6, Rosa26-Cre $e^{\text {ERT2 }}, I_{d 2}{ }^{\text {GFP-knockin }}$, and UbcGFP mice were obtained from The Jackson Laboratory. Kdr (Vegfr $)^{t / f l l}$, $K D R^{G F P-k n o c k i n}, R o s a 26-C r e^{E R T 2}, K d r^{f / f l}\left(\operatorname{Veg} f r^{f / f f}\right)$, and Ubc-GFP mice on a C57BL/6 background were obtained from Shahin Rafii. Littermate controls were used in all animal experiments. Rosa26-Cre ${ }^{\text {ERT2 }}$ mice were crossed with $K d r^{f / f l}$ mice to generate Rosa26-Cre ${ }^{E R T 2} K d r^{A / f l}$ mice. The littermates of Rosa26-Cre ${ }^{E R T 2} K d r^{t /+}$ mice served as controls. GL261 cells were obtained from David Zagzag (Department of Pathology, New York University, New York, New York, USA). HCMEC cells were obtained from Babette Weksler (Department of Medicine, Weill Cornell Medicine).

RCAS/TVA glioma model and symptom-free survival analysis. RCAS- $h P D G F b-H A-S V 4 O$ containing human PDGFb with partially deleted 59UTR, RCAS-hPDGFb-HA, and RCAS-Cre vectors were transfected into DF-1 chicken cells (ATCC). DF-1 cells transfected with RCAS-PSG were cultured in 10\% FBS-DMEM media under standard conditions. For tumor induction, transfected DF-1 cells were trypsinized, centrifuged, and injected into the brain parenchyma of $n$-tva mice on a heterozygous Ink4a/Arft/-Pten ${ }^{f / / l}$ background (obtained from Eric Holland) at P0-P2. The mice transgenically expressed the RCAS-binding tva receptor after the nestin promoter. For BMT study, the RCAS/TVA mice at week 2 were subject to 9.5 Gy irradiation with their heads shielded and transplantation of donor BM cells through retroorbital injection as well as the procedures described in the BMT section. Upon appearance of brain tumor symptoms (e.g., poor grooming, lethargy, macrocephaly, hydrocephaly, hemiparesis, substantial weight loss), mice were anesthetized by intraperitoneal injection of $1 \mathrm{mg} / \mathrm{kg}$ Nembutal solution or a ketamine $(150 \mathrm{mg} / \mathrm{kg}) / x y l a z i n e ~(15$ $\mathrm{mg} / \mathrm{kg}$ ) cocktail and underwent transcardiac perfusion with $10 \mathrm{ml}$ icecold heparin in normal saline, followed by $10 \mathrm{ml}$ ice-cold $4 \%$ paraformaldehyde. Brain tissue was extracted and postfixed for 30 minutes in ice-cold $4 \%$ paraformaldehyde and transferred to $30 \%$ sucrose at $4{ }^{\circ} \mathrm{C}$ for cryoprotection. For a subset of animals sacrificed using $\mathrm{CO}_{2}$, brain tissue was extracted and fixed for 3 days in $4 \%$ paraformaldehyde and subsequently processed using standard paraffin embedding and processing. Kaplan-Meier curves for symptom-free survival were made using Prism software and analyzed with a standard log-rank test. The end-point was defined as the time mice had developed symptoms such as hydrocephaly and lethargy.

Genotyping. Genotyping methods, primer sequences, and PCR conditions were as follows: Rosa26-Cre ${ }^{E R T 2}$ : WT forward, 5'-AAAGTCGCTCTGAGTTGTTAT-3', WT reverse, 5'-GGAGCGGGAGAAATGGATATG-3', mutant reverse, 5'-CCTGATCCTGGCAATTTCG-3', $94 \times$ 30 seconds, $65 \times 30$ seconds, $72 \times 60$ seconds, 35 cycles, WT band 650 bp, mutant band 825; Kdrf/fl: forward, 5'-CCACAGAACAACTCAGGGCTA-3', reverse, 5'-GGGAGCAAAGTCTCTGGAAA-3', $94 \times 30$ seconds, $60 \times 30$ seconds, $72 \times 60$ seconds, 35 cycles, WT band $185 \mathrm{bp}$, with LoxP site 231 bp; Id ${ }^{\text {GFP-Knockin }}$ : primer 12341, 5'-CTTCCTCCTACGAGCAGCAT-3', primer 12342, 5'-CTCACCTGCAAGGACAGGAT-3', primer 12343, 5'-GCTCCTGGACAGGAATCAAG-3', $94 \times 30$ seconds, $59 \times 60$ seconds, $72 \times 60$ seconds, 35 cycles, WT band $371 \mathrm{bp}$, mutant band 750 bp; GFP: forward, 5'-CTGCTGCCCGACAACCA-3', reverse, 5'-TCCAGCAGGACCATGTGATC-3'.

Intracranial injections and MRI/bioluminescence imaging. Luciferaselabeled GL261 cells $\left(1 \times 10^{5}\right)$ were stereotactically inoculated into the right hemispheres of 8-week-old mouse brains $(1.5 \mathrm{~mm}$ posterior to the bregma and $1.5 \mathrm{~mm}$ to the right of the sagittal suture to a depth of 2.5 mm below the surface of the skull using a $1 \mu \mathrm{l}$ Hamilton syringe). Mice were euthanized when they presented with neurological symptoms or substantial weight loss. Mice were examined and weighed daily. Bioluminesence and MRI were performed to monitor the progression of tumors. Bioluminesence was performed under the IVIS-100 (Xenogen) system, and images were acquired and analyzed by Living Image (Caliper). High-resolution MRI images were acquired on a 7.0-T animal scanner system (Bruker Biospin). $200 \mu \mathrm{l}$ of $40 \mathrm{mM} \mathrm{Gd-DTPA}$ or Gd-albumin was injected by tail vein to measure brain tumor contrast enhancement. During imaging, animals were under isoflurane anesthesia and monitored for respiration and heart rate; the temperature was kept at $37^{\circ} \mathrm{C}$. Images were analyzed by an OsiriX DICOM viewer.

Tamoxifen administration. To induce KDR gene ablation, at day 3 after injection, mice with intracranially implanted tumor cells were treated with tamoxifen $(20 \mathrm{mg} / \mathrm{kg}$ intraperitoneally) for 6 days, interrupted for 3 days after the third dose. After 3 days of respite, the fourth dose was reinstituted for an additional 3 days, resulting in

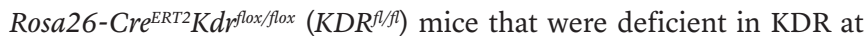
both alleles. The control animals were Rosa26-CreERT2 $\mathrm{Kdr} \mathrm{r}^{\mathrm{fl}+}$ mice.

$B M T$ and competitive BMT. Recipient mice were lethally irradiated with a single dose of $9.5 \mathrm{~Gy}$ applied to their whole bodies with their heads lead shielded; 24 hours after irradiation, $5 \times 10^{6}$ total BM cells were injected by tail vein. The BM of donor mice was harvested by flushing femurs and tibias with cold sterile EDTA/PBS. Engraftment was confirmed by complete blood count using a Hemavet 850 blood cell counter (Drew Scientific) and flow cytometry to ensure adequate hematopoiesis. For competitive BMT, we mixed donor BM cells from Ubc-GFP Rosa26-Cre $e^{E R T 2}$ $K d r^{f / f l}$ mice and Rosa26-Cre ${ }^{E R T 2} K d r^{f /+}$ mice at a 1:1 ratio and injected 5 $\times 10^{6}$ total cells into recipient mice; 3 days after tumor implantation, we treated mice with tamoxifen to induce the deletion of the $K d r$ allele.

IHC and immunostaining. Antibodies utilized for IHC and immunostaining included the following: anti-mouse CD11b (clone: M1/70, eBioscience), anti-human CD11b (catalog ab52478, Abcam), anti-VEGFR2 (KDR) (catalog 9698, Cell Signaling), anti-collagen IV (catalog ab19808, Abcam), anti-CD31 (clone: MEC13.3, BD Biosciences), anti-von Willebrand factor (catalog AB7356, Millipore); and antiKi67 (catalog ab15580, Abcam).

Secondary antibodies utilized included the following: FITC- and Cy3-conjugated donkey anti-goat/ anti-rabbit/anti-rat (catalog 711-095152; 711-165-152; 712-095-150; 712-165-150, Jackson ImmunoResearch); Alexa Fluor 488- and Alexa Fluor 555-conjugated donkey anti-mouse (catalog A-21202; A-31570; Invitrogen); and biotinylated goat anti-rabbit (catalog BA-1000, Vector).

Cell preparation and flow cytometry (intracellular staining). Hematopoietic cells isolated from buffy coats were subjected to trypan blue staining or live/dead cell staining (Fixable Dead Cell Stain Kits, Invitrogen). Cells were then fixed in $0.05 \%$ formaldehyde for 10 to $15 \mathrm{~min}$ utes followed by disruption of membrane by detergent Tween 20 at $0.5 \% \mathrm{v} / \mathrm{v}$ in PBS for 15 minutes. Cells were washed by PBS (containing $0.1 \%$ Triton $\mathrm{X}-100$ or other permeabilizing detergent) and centrifuged at $300 \mathrm{~g}$ for 5 minutes; supernatant was discarded. Cells were resuspended in the volume remaining. Antibodies were given according to manufacturer directions. Antibodies were prepared in permeabilization buffer to ensure that cells remained permeable. Cells were counted, and $1 \times 10^{6}$ cells from each sample were subject to flow analysis using FACSCalibur (BD). All cells were stained at $4^{\circ} \mathrm{C}$ in PBS with $2 \%$ FCS. The following antibodies were used for staining: APC anti- 
human KDR/isotype control, PEcy7-Ly6C, PE-Ly6G, PE-CD33, Biotin Mouse Lineage Panel (559971, BD Biosciences - Pharmingen), FITC/eF450 CD45 (clone 30-F11), PE/APC-c-Kit (clone 2B8), PE/ PEcy7-Sca-1 (clone D7), FITC/eF450-CD11b (clone M1/70), PE-Gr-1 (clone RB6-8C5), PEcy7-HLA-DR (clone LN3), AF-700/APC-CD16 (clone CB16), APC/PEcy7-CD14 (clone 61D3), eF5.5-FcrRII (AT1302), Pacific Blue/FITC-streptavidin (11-4317,eBioscience), and Pacific Blue/Mouse Lineage Panel (133310, BioLegend). Data were analyzed by FlowJo software. Analysis was done within single cell gate after the exclusion of debris and dead cells.

RNA sequencing analysis. $\mathrm{CD} 11 \mathrm{~b}^{+} \mathrm{Gr}-1^{+} \mathrm{KDR}-\mathrm{GFP}^{+}$myeloid cells were sorted from peripheral blood, and c-Kit ${ }^{+} \mathrm{Sca}-\mathrm{1}^{-} \mathrm{KDR}-\mathrm{GFP}^{+}$progenitor cells were sorted from BM cells by FACS (BD) directly into cell lysis buffer provided in the RNeasy Micro Kit (QIAGEN). Total RNA $\mathrm{BM}$ progenitor cells in each group were extracted with an RNeasy Microkit followed by cDNA amplification; 3 or 4 independent samples from each group were subjected to RNA-sequencing (Illumina HiSeq 2000). Gene expression data were analyzed using the Limma package in $\mathrm{R} /$ Bioconductor software, using a $P$ value of less than 0.001 and fold change greater than 2 . By unsupervised analysis, these samples were clustered based on their expression profiles. $Z$ scores were used in the cluster analysis. All original microarray data were deposited in the NCBI's Gene Expression Omnibus (GEO GSE94271).

In vitro culture of HPCs. Whole BM was harvested from 7- to 10 -week-old donor mice (C57BL/6 or $\mathrm{Kdr} / \mathrm{Id} 2$ deficient mice as specified in each experiment). Briefly, femur and tibia were removed and the BM was harvested by flushing it with cold RPMI medium containing $1 \%$ penicillin/streptomycin using a syringe equipped with a 25 -gauge needle. Uncommitted BM progenitors, highly enriched in HSCs, were negatively selected using either the Lineage Cell Depletion Kit for mouse (Miltenyi Biotec) or the Hematopoietic Progenitor Enrichment Kit (StemCell Technologies) following the manufacturer's protocols. The lineage ${ }^{-}$selected cells ( Lin $^{-}$BM cells) were placed in culture at $1 \times$ $10^{6}$ cells $/ \mathrm{ml}$ in StemSpan medium (StemCell Technologies) containing $1 \%$ penicillin/streptomycin, $100 \mathrm{ng} / \mathrm{ml}$ murine stem cell factor (SCF), $100 \mathrm{ng} / \mathrm{ml}$ human Fms-like tyrosine kinase 3, $100 \mathrm{ng} / \mathrm{ml}$ human IL-11, and $20 \mathrm{ng} / \mathrm{ml}$ murine IL-3. All cytokines were purchased from PeproTech. The Lin $^{-} \mathrm{BM}$ cells were transduced with the appropriate lentiviral particles at an MOI of 10 . At 18 to 24 hours after transduction, the transduced Lin $^{-} \mathrm{BM}$ cells were harvested, washed, and resuspended in RPMI medium containing $1 \%$ penicillin/streptomycin at $2.5 \times 10^{6}$ cells/ $\mathrm{ml}$ for subsequent studies. For treatment of tumor cells, conditioned medium, serum-free RPMI that had been conditioned by GL261 tumor cells for 24 hours, was added at a 1:1 ratio into Lin $^{-}$BM cells. For treatment of cytokines or antibodies, $1.5 \mathrm{ng} / \mathrm{ml} \mathrm{TGF-} \beta 1,10 \mathrm{ng} / \mathrm{ml} \mathrm{GM-CSF}$, or $10 \mu \mathrm{g} / \mathrm{ml}$ TGF- $\beta 1$ neutralized antibodies were used.

$R T-P C R$. RNA from the sorted or cultured cells was extracted using the RNeasy kit (QIAGEN) and reverse transcribed using Superscript III reverse transcriptase (Invitrogen). Quantitative PCR (QPCR) was performed on a 7500 Fast Real Time PCR System (Applied Biosystems) with SYBR green (Life Technologies). Probes were as follows: murine Kdr: 5'-CCATTGGAGGAACCAGAAGT-3', 5'-CTCTTCTGATGCAAGGACCA-3'; human ID2: 5'-TCAGCCTGCATCACCAGAGA-3', 5'-CTGCAAGGACAGGATGCTGATA-3'; murine Id2: 5'-AAAACAGCCTGTCGGACCAC-3'， 5'-CTGGGCACCAGTTCCTTGAG-3'; murine Cd11b: 5'-CTGAGAAATGACGGTGAGGA-3', 5'-CAGCAGGCTTTACAAACCAA-3'; murine CD68: 5'-GCGGTGGAATACAAT-
GTGTC-3', 5'-AGAGATGAATTCTGCGCCAT-3'; murine c-Kit: 5'-AGAAGGAAGCGTGACTCGTT-3', 5'-TTGAACTGTCACAGGAAGGC-3'; murine $\beta$-actin: 5 '-CGGTTGGCCTTAGGGTTCA-3', 5'-GTGGGCCGCTCTAGGCACCA-3'.

CFU assay. BM cells $\left(1 \times 10^{4}\right)$ from each group of mice were harvested and cultured in 2 wells of a 24 -well culture plate supplemented with standard methylcellulose medium containing $10 \mathrm{ng} / \mathrm{ml}$ murine IL-3, $10 \mathrm{ng} / \mathrm{ml}$ human IL-6, and $50 \mathrm{ng} / \mathrm{ml}$ murine SCF (StemCell Technologies). Granulocyte, macrophage, and granulocyte-macrophage CFU were counted after 7 days of culture.

Endothelial tubule formation. GFP-labeled HCMEC/D3 $\left(5 \times 10^{4}\right.$ / well) cells were seeded on 24-well plates coated by Matrigel with reduced growth factors ( $200 \mu \mathrm{l} /$ well, BD Biosciences) and incubated for 6 hours followed by coculture with BMDCs. Spatial organization and sprout configuration were assessed after 12 hours using in situ fluorescence imaging. Cumulative sprout lengths of tubules represented by area of $\mathrm{GFP}^{+}$field were quantitatively analyzed using Image (NIH).

Matrigel plugs. Growth factors were thawed and Matrigel (BD Biosciences) was reduced on ice overnight at $4^{\circ} \mathrm{C}$. The Matrigel was mixed with specified BMDCs on a vortex and placed on ice. A syringe with each solution was used to inject 0.5 or $1 \mathrm{ml}$ subcutaneously into the ventral area of each mouse. After 10 days, mice were injected with $100 \mu \mathrm{l}$ $(10 \mathrm{mg} / \mathrm{ml})$ 70-kD DsRed-dextran by tail vein. Ten minutes later, animals were sacrificed, followed by $4 \%$ paraformaldehyde perfusion, and plugs removed. Plugs appeared as bumps on the ventral side of the animal. Whole plugs were placed in gradients of methanol from $25 \%$ to $100 \%$ and then into benzyl alcohol benzyl benzoate (Sigma-Aldrich) at a 1:2 ratio. Plugs were then mounted for fluorescent imaging.

Zymography. Supernatant was harvested from specified cells, concentrated with 10-kD centrifugal filter columns (Millipore), and loaded without a reducing reagent to $12 \%$ gelatin gel (Invitrogen). Zymography development was done with Novex precast zymography buffers (Invitrogen) by following the manufacturer's instructions. Following electrophoresis, the gel was incubated with $100 \mathrm{ml}$ of renaturing buffer under gentle agitation for 30 minutes. Renaturing buffer was removed, $100 \mathrm{ml}$ of developing buffer was added, and the gel was incubated under agitation for 30 minutes. Developing buffer was then removed and another $100 \mathrm{ml}$ of developing buffer was added. The zymogram was then placed in the humidified incubator set to $37^{\circ} \mathrm{C}$ and incubated overnight. Developing buffer was removed and replaced with $100 \mathrm{ml}$ of deionized water. The zymogram was washed 3 times under gentle agitation for 5 minutes, stained with $0.5 \%$ Coomassie G250 in 30\% ethanol and $10 \%$ acetic acid for 30 minutes, and then destained in $30 \%$ ethanol $/ 10 \%$ acetic acid. Destaining was quenched by transferring the gel to $2 \%$ acetic acid when the clear bands were observed.

CHIP assay. BM progenitor cells were collected and cultured as described above. Protein-DNA complexes were crosslinked by incubating the cells with $37 \%$ formaldehyde diluted to a $1 \%$ final concentration and incubating with cells on a rocking or shaking device for 15 minutes at room temperature. Formaldehyde was quenched by adding $1 \mathrm{M}$ glycine diluted to a final concentration of $125 \mathrm{mM}$. Cells were rocked for 5 minutes at room temperature and pelleted; medium was removed. Protease inhibitors were added to the Lysis buffer (10 $\mu \mathrm{g} / \mathrm{ml}$ leupeptin, $10 \mu \mathrm{g} / \mathrm{ml}$ aprotinin, and $1 \mathrm{mM}$ PMSF). The cell pellet was resuspended in $500 \mu$ of lysis buffer per $5 \times 10^{6}$ cells and incubated on ice for 10 minutes. Samples were sonicated to shear chromatin to an average length of about $1 \mathrm{~kb} .500 \mu \mathrm{l}$ of each 
sample was transferred to a $1.5-\mathrm{ml}$ microcentrifuge tube and centrifuged for 10 minutes using a refrigerated ultracentrifuge at 12,000 $g$. Supernatant was collected in a clean tube and the pellet discarded. Supernatant was diluted by adding $1 \mathrm{ml}$ of dilution buffer and $5 \mu \mathrm{g}$ of the E2A antibody (Santa Cruz Biotechnology Inc., sc-763) or normal IgG to the samples and incubated overnight at $4^{\circ} \mathrm{C}$ on a rotating device. Secondary biotinylated antibody was added at $5 \mu \mathrm{g}$ and incubated for 1 hour at $4^{\circ} \mathrm{C}$ on a rotating device. We added 100 $\mu \mathrm{l}$ of streptavidin magnetic beads to the samples and rotated for 30 minutes at $4^{\circ} \mathrm{C}$. The beads were collected by leaving the tube in the magnet for 2 minutes and were washed with prechilled wash buffers. After the last wash, $100 \mu \mathrm{l}$ of chelating resin solution was added directly to the beads and pipetted up and down for about $10 \mathrm{sec}-$ onds. The sample was boiled for 10 minutes using a heat block or a temperature-controlled water bath and microcentrifuged at 12,000 $g$ for 1 minute at room temperature before transferring the supernatant to a clean microcentrifuge tube. The DNA preparation was cleaned and concentrated using a DNA purification kit (QIAGEN) and resuspended in $30 \mu \mathrm{l}$ of deionized or distilled water. $2 \mu \mathrm{l}$ of the DNA sample was used in the real-time PCR reactions.

The probes for KDR promoter region were as follows: sense: CCTGGTTTGCCACCTGCTTCTG; antisense, TGTAACATGGTGCTTTTCTTT.

Statistics. All data are given as mean \pm SD or \pm SEM. $n$ was used to show the number of biological replicates within figure legends. (a) For quantification of IHC, $n$ represents the number of biological samples within each group. First, we randomly selected 5 slides from each sample/mouse. Nine fields from each slide were counted, and an average number per field was used to represent each slide. Then the mean of values from 5 slides was used to represent each sample/ mouse and graphs were generated based on $n$ independent samples/ mouse. Two independent observers blindly measured or counted to determine intra- and interobserver variability. (b) $n$ in animal experiments represents the number of mice in each group. (c) $n$ in RT-PCR and cell count experiments represents the number of independent wells of treatment per condition, each of which represents the mean value from 3 experimental wells. In "Endothelial tubule formation," the value of each well is the mean of cell counts of six $100 \times$ fields randomly chosen within a well, and $n$ is the number of independent wells of treatment per condition. Differences between the mean parameter values derived from 2 groups were compared using 2-tailed Student's $t$ tests and 1-way ANOVA, followed by a post-hoc test for comparison of multiple groups. Significance was determined as a $P$ value of less than 0.05 .

Study approval. All animal experiments were performed according to the guidelines set forth by the Institutional Animal Care and Use Committee at Weill Cornell Medicine. Human peripheral blood samples were obtained from subjects with grades I, II, III, and IV gliomas at Weill Cornell Medical College and were histologically confirmed as gliomas. Subjects with grade II gliomas were followed up over 12 months. Tumor progression was determined by either histology or radiography. All individuals provided informed consent for blood donation according to a protocol approved by the institutional review board.

\section{Author contributions}

YH, JB, DL, and JG designed the study. YH, PR, CH, JHK, EG, WC, $\mathrm{EV}$, and RX conducted experiments. $\mathrm{YH}, \mathrm{WH}, \mathrm{TZ}$, and JX acquired data. YH, WH, TZ, HP, JB, BD, SR, DL, and JG analyzed data. DP, $\mathrm{JH}, \mathrm{EH}, \mathrm{BD}$, and SR provided important materials. $\mathrm{YH}, \mathrm{PR}, \mathrm{CH}$, BC, RY, IM, DL, and JG wrote the manuscript.

\section{Acknowledgments}

This study was supported by the Department of Defense Congressionally Directed Medical Research Programs (DOD CDMRP, CA120318 to Y. Huang), Elizabeth's Hope (J. Greenfield), the Starr Foundation, the Paduano Foundation, the Champalimaud Foundation, the Malcolm Hewitt Wiener Foundation, the POETIC Foundation, the Sohn Foundation, the Hartwell Foundation, and the Children's Cancer and Blood Foundation (all to D. Lyden).

Address correspondence to: David Lyden, Department of Pediatrics, Weill Medical Medicine, 413 E. 69th Street, Box 284, New York, New York 10021, USA. Phone: 646.962.6238; E-mail: dcl2001@med.cornell.edu. Or to: Jeffrey P. Greenfield, Department of Neurological Surgery, Weill Cornell Medicine, 525 E 68th Street, Box 99, New York, New York 10065, USA. Phone: 212.746.2363; E-mail:.jpgreenf@med.cornell.edu.

HP's present address is: Microenvironment and Metastasis Group, Department of Molecular Oncology, Spanish National Cancer Research Center (CNIO), Madrid, Spain.
1. Joyce JA, Pollard JW. Microenvironmental regulation of metastasis. Nat Rev Cancer. 2009;9(4):239-252.

2. Kaplan RN, et al. VEGFR1-positive haematopoietic bone marrow progenitors initiate the pre-metastatic niche. Nature. 2005;438(7069):820-827.

3. Kessenbrock K, Plaks V, Werb Z. Matrix metalloproteinases: regulators of the tumor microenvironment. Cell. 2010;141(1):52-67.

4. Qian BZ, et al. CCL2 recruits inflammatory monocytes to facilitate breast-tumour metastasis. Nature. 2011;475(7355):222-225.

5. Du R, et al. HIF1alpha induces the recruitment of bone marrow-derived vascular modulatory cells to regulate tumor angiogenesis and invasion. Cancer Cell. 2008;13(3):206-220.
6. Gabrilovich DI, Ostrand-Rosenberg S, Bronte V. Coordinated regulation of myeloid cells by tumours. Nat Rev Immunol. 2012;12(4):253-268.

7. Peinado H, et al. Melanoma exosomes educate bone marrow progenitor cells toward a prometastatic phenotype through MET. Nat Med. 2012;18(6):883-891.

8. Kioi M, Vogel H, Schultz G, Hoffman RM, Harsh GR, Brown JM. Inhibition of vasculogenesis, but not angiogenesis, prevents the recurrence of glioblastoma after irradiation in mice. JClin Invest. 2010;120(3):694-705.

9. Louis DN, et al. The 2007 WHO classification of tumours of the central nervous system. Acta Neuropathol. 2007;114(2):97-109.

10. Huse JT, Holland EC. Targeting brain cancer: advances in the molecular pathology of malig- nant glioma and medulloblastoma. Nat Rev Cancer. 2010;10(5):319-331.

11. Charles NA, Holland EC, Gilbertson R, Glass R, Kettenmann $\mathrm{H}$. The brain tumor microenvironment. Glia. 2011;59(8):1169-1180.

12. Kim JH, Huse JT, Huang Y, Lyden D, Greenfield JP. Molecular diagnostics in paediatric glial tumours. Lancet Oncol. 2013;14(1):e19-e27.

13. Bourne TD, Schiff D. Update on molecular findings, management and outcome in low-grade gliomas. Nat Rev Neurol. 2010;6(12):695-701.

14. Stupp R, et al. Radiotherapy plus concomitant and adjuvant temozolomide for glioblastoma. N Engl J Med. 2005;352(10):987-996.

15. Wen PY, Kesari S. Malignant gliomas in adults. N Engl JMed. 2008;359(5):492-507.

16. Lyden D, et al. Impaired recruitment of 
bone-marrow-derived endothelial and hematopoietic precursor cells blocks tumor angiogenesis and growth. Nat Med. 2001;7(11):1194-1201.

17. Rafii S, Lyden D, Benezra R, Hattori K, Heissig B. Vascular and haematopoietic stem cells: novel targets for anti-angiogenesis therapy? Nat Rev Cancer. 2002;2(11):826-835.

18. Greenfield JP, et al. Surrogate markers predict angiogenic potential and survival in patients with glioblastoma multiforme. Neurosurgery. 2009;64(5):819-826.

19. Agudo J, et al. The miR-126-VEGFR2 axis controls the innate response to pathogen-associated nucleic acids. Nat Immunol. 2014;15(1):54-62.

20. Fomchenko EI, et al. Recruited cells can become transformed and overtake PDGF-induced murine gliomas in vivo during tumor progression. PLoS One. 2011;6(7):e20605

21. Shih AH, Dai C, Hu X, Rosenblum MK, Koutcher JA, Holland EC. Dose-dependent effects of platelet-derived growth factor-B on glial tumorigenesis. Cancer Res. 2004;64(14):4783-4789.

22. Wong KL, et al. Gene expression profiling reveals the defining features of the classical, intermediate, and nonclassical human monocyte subsets. Blood.2011;118(5):e16-e31.

23. Kee BL. E and ID proteins branch out. Nat Rev Immunol. 2009;9(3):175-184.

24. Fridlender ZG, et al. Polarization of tumorassociated neutrophil phenotype by TGF-beta: "N1" versus “N2" TAN. Cancer Cell. 2009;16(3):183-194.

25. Shojaei F, et al. Tumor refractoriness to anti-VEGF treatment is mediated by $\mathrm{CD} 11 \mathrm{~b}+\mathrm{Gr} 1+$ myeloid cells. Nat Biotechnol. 2007;25(8):911-920.

26. Ostrand-Rosenberg S, Sinha P. Myeloid-derived suppressor cells: linking inflammation and cancer. J Immunol. 2009;182(8):4499-4506.

27. Nozawa H, Chiu C, Hanahan D. Infiltrating neutrophils mediate the initial angiogenic switch in a mouse model of multistage carcinogenesis. Proc Natl Acad Sci USA. 2006;103(33):12493-12498.

28. Eichmann A, Simons M. VEGF signaling inside vascular endothelial cells and beyond. Curr Opin Cell Biol. 2012;24(2):188-193.

29. Heissig B, et al. Recruitment of stem and progenitor cells from the bone marrow niche requires
MMP-9 mediated release of kit-ligand. Cell. 2002;109(5):625-637.

30. Rey S, et al. Synergistic effect of HIF-1alpha gene therapy and HIF-1-activated bone marrowderived angiogenic cells in a mouse model of limb ischemia. Proc Natl Acad Sci USA. 2009;106(48):20399-20404.

31. Lewis CE, De Palma M, Naldini L. Tie2expressing monocytes and tumor angiogenesis: regulation by hypoxia and angiopoietin-2. Cancer Res. 2007;67(18):8429-8432.

32. Rafii S, Lyden D. Therapeutic stem and progenitor cell transplantation for organ vascularization and regeneration. Nat Med. 2003;9(6):702-712.

33. Avecilla ST, et al. Chemokine-mediated interaction of hematopoietic progenitors with the bone marrow vascular niche is required for thrombopoiesis. Nat Med. 2004;10(1):64-71.

34. Hattori K, et al. Activation of VEGF receptor-1 (VEGFR1, Flt-1) expressed on hematopoietic stem cells promotes cell motility and is essential for marrow reconstitution. Blood. 2001;98(11):710A-711A.

35. Gordon S, Taylor PR. Monocyte and macrophage heterogeneity. Nat Rev Immunol. 2005;5(12):953-964.

36. Pyonteck SM, et al. CSF-1R inhibition alters macrophage polarization and blocks glioma progression. Nat Med. 2013;19(10):1264-1272.

37. Gabrilovich DI, Nagaraj S. Myeloid-derived suppressor cells as regulators of the immune system. Nat Rev Immunol. 2009;9(3):162-174.

38. Lampugnani MG, Orsenigo F, Gagliani MC, Tacchetti C, Dejana E. Vascular endothelial cadherin controls VEGFR-2 internalization and signaling from intracellular compartments. J Cell Biol. 2006;174(4):593-604.

39. Santos SC, Dias S. Internal and external autocrine VEGF/KDR loops regulate survival of subsets of acute leukemia through distinct signaling pathways. Blood. 2004;103(10):3883-3889.

40. Lanahan AA, et al. VEGF receptor 2 endocytic trafficking regulates arterial morphogenesis. Dev Cell. 2010;18(5):713-724.

41. Domingues I, Rino J, Demmers JA, de Lanerolle
P, Santos SC. VEGFR2 translocates to the nucleus to regulate its own transcription. PLoS One. 2011;6(9):e25668.

42. Santos SC, et al. VEGF and VEGFR-2 (KDR) internalization is required for endothelial recovery during wound healing. Exp Cell Res. 2007;313(8):1561-1574.

43. Stockmann C, et al. Deletion of vascular endothelial growth factor in myeloid cells accelerates tumorigenesis. Nature. 2008;456(7223):814-818.

44. Murray PJ, Wynn TA. Protective and pathogenic functions of macrophage subsets. Nat Rev Immunol. 2011;11(11):723-737.

45. Sica A, Bronte V. Altered macrophage differentiation and immune dysfunction in tumor development. JClin Invest. 2007;117(5):1155-1166.

46. De Palma M, et al. Tie2 identifies a hematopoietic lineage of proangiogenic monocytes required for tumor vessel formation and a mesenchymal population of pericyte progenitors. Cancer Cell. 2005;8(3):211-226.

47. Swann JB, Smyth MJ. Immune surveillance of tumors. JClin Invest. 2007;117(5):1137-1146.

48. Bingle L, Brown NJ, Lewis CE. The role of tumour-associated macrophages in tumour progression: implications for new anticancer therapies. J Pathol. 2002;196(3):254-265.

49. Leek RD, Hunt NC, Landers RJ, Lewis CE, Royds JA, Harris AL. Macrophage infiltration is associated with VEGF and EGFR expression in breast cancer. J Pathol. 2000;190(4):430-436.

50. Steidl C, et al. Tumor-associated macrophages and survival in classic Hodgkin's lymphoma. N Engl JMed. 2010;362(10):875-885.

51. Cochrane SW, Zhao Y, Welner RS, Sun XH. Balance between Id and $\mathrm{E}$ proteins regulates myeloid-versus-lymphoid lineage decisions. Blood. 2009;113(5):1016-1026.

52. Mercer EM, et al. Multilineage priming of enhancer repertoires precedes commitment to the $\mathrm{B}$ and myeloid cell lineages in hematopoietic progenitors. Immunity. 2011;35(3):413-425.

53. Li H, Ji M, Klarmann KD, Keller JR. Repression of Id 2 expression by Gfi- 1 is required for B-cell and myeloid development. Blood. 2010;116(7):1060-1069. 